Gill's model of the Antarctic Circumpolar Current, revisited: the role of latitudinal variations in wind stress

David P. Marshall, David R. Munday, Lesley C. Allison, Russell J. Hay, Helen L. Johnson

PII: S1463-5003(15)00234-6

DOI: 10.1016/j.ocemod.2015.11.010

Reference: $\quad$ OCEMOD 1054

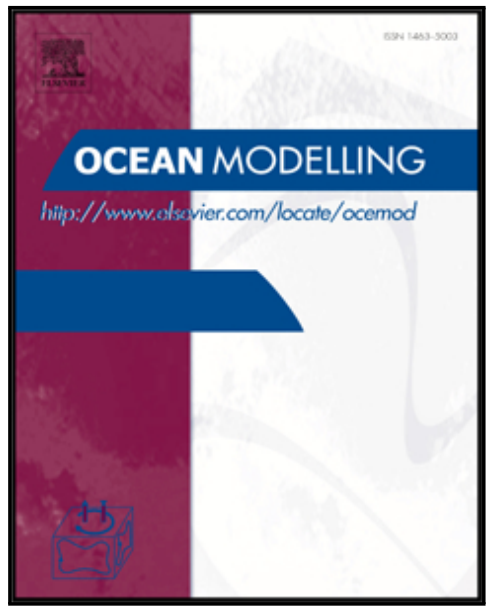

To appear in:

Ocean Modelling

Received date:

25 July 2015

Revised date:

5 November 2015

Accepted date:

19 November 2015

Please cite this article as: David P. Marshall, David R. Munday, Lesley C. Allison, Russell J. Hay, Helen L. Johnson, Gill's model of the Antarctic Circumpolar Current, revisited: the role of latitudinal variations in wind stress, Ocean Modelling (2015), doi: 10.1016/j.ocemod.2015.11.010

This is a PDF file of an unedited manuscript that has been accepted for publication. As a service to our customers we are providing this early version of the manuscript. The manuscript will undergo copyediting, typesetting, and review of the resulting proof before it is published in its final form. Please note that during the production process errors may be discovered which could affect the content, and all legal disclaimers that apply to the journal pertain. 


\section{Highlights}

- Adrian Gill's model of the Antarctic Circumpolar Current (ACC) is reinterpreted.

- Eddy-induced vortex stretching plays a key role in the vorticity balance of the ACC.

- A substantial ACC remains when the wind is $3000 \mathrm{~km}$ north of Drake Passage.

- The net wind stress over the ACC streamlines is a predictor of its volume transport.

- A correction for basin-wide pressure gradients leads to good quantitative agreement. 


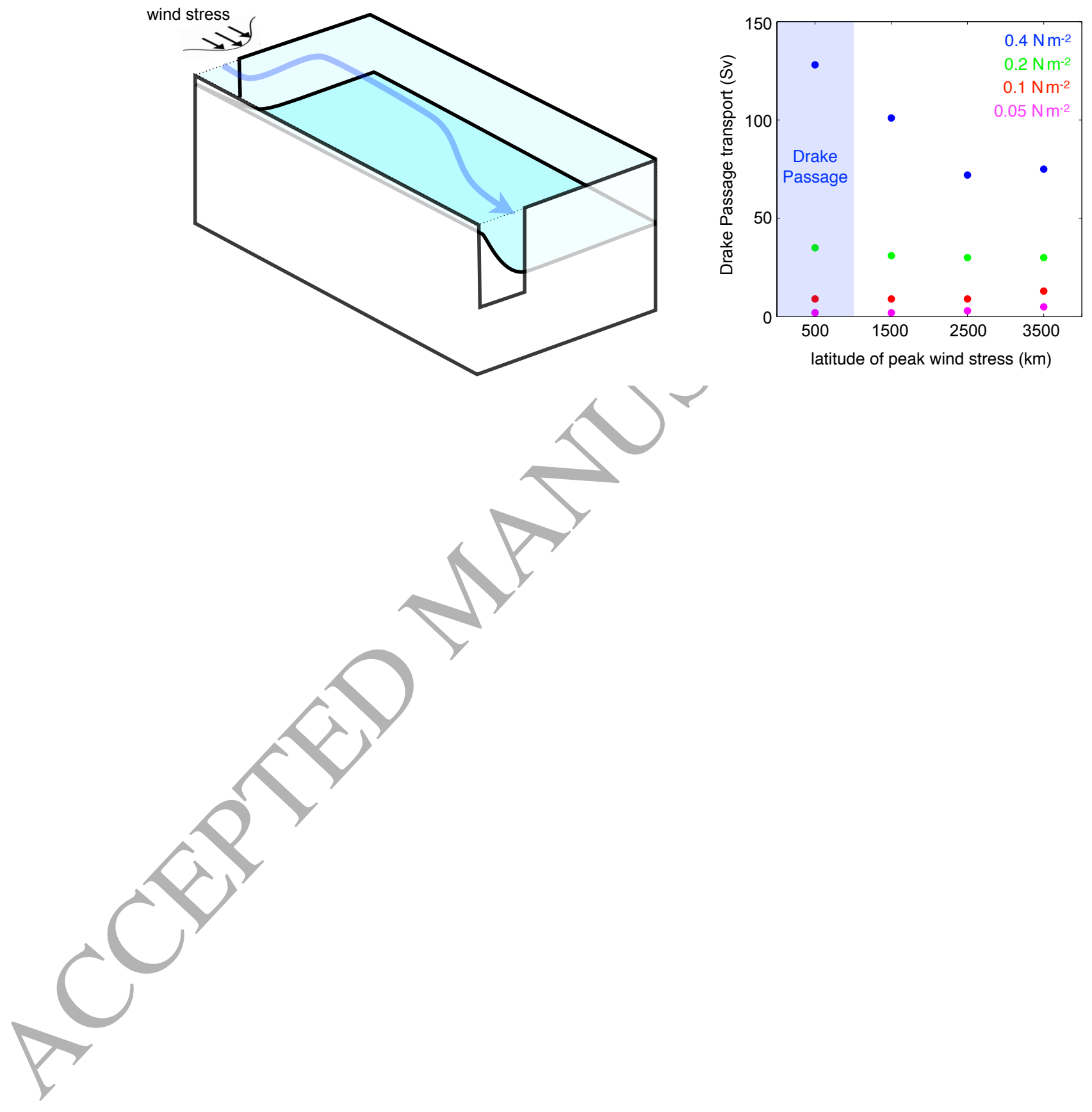




\title{
Gill's model of the Antarctic Circumpolar Current, revisited: the role of latitudinal variations in wind stress
}

\author{
David P. Marshall ${ }^{\mathrm{a}, *}$, David R. Munday ${ }^{\mathrm{a}, \mathrm{b}}$, Lesley C. Allison ${ }^{\mathrm{c}}$, Russell J. Hay ${ }^{\mathrm{d}}$, \\ Helen L. Johnson ${ }^{\mathrm{e}}$ \\ ${ }^{a}$ Department of Physics, University of Oxford, Oxford, OX1 3PU, United Kingdom \\ ${ }^{b}$ British Antarctic Survey, Cambridge, CB3 OET, United Kingdom \\ ${ }^{c}$ Met Office, Exeter, EX1 3PB, United Kingdom \\ ${ }^{d}$ Department of Physics, Yale University, United States \\ ${ }^{e}$ Department of Earth Sciences, University of Oxford, Oxford, OX1 3AN, United Kingdom
}

\begin{abstract}
Adrian Gill's (1968) model of the Antarctic Circumpolar Current (ACC) is reinterpreted for a stratified, reduced-gravity ocean, where the barotropic streamfunction is replaced by the pycnocline depth, and the bottom drag coefficient by the Gent and McWilliams eddy diffusivity. The resultant model gives a simple description of the lateral structure of the ACC that is consistent with contemporary descriptions of ACC dynamics. The model is used to investigate and interpret the sensitivity of the ACC to the latitudinal profile of the surface wind stress. A substantial ACC remains when the wind jet is shifted north of the model Drake Passage, even by several thousand kilometers. The integral of the wind stress over the circumpolar streamlines is found to be a useful predictor of the magnitude of the volume transport through the model Drake Passage, although it is necessary to correct for basin-wide zonal pressure gradients in order to obtain good quantitative agreement.
\end{abstract}

Keywords: Antarctic Circumpolar Current, ocean circulation, geostrophic eddies, wind stress, Drake Passage

\section{Introduction}

The Antarctic Circumpolar Current (ACC) is the only current to circumnavigate the globe, with a thermal wind volume transport through Drake Passage of $4 \quad 137 \pm 7 \mathrm{~Sv}\left(1 \mathrm{~Sv} \equiv 10^{6} \mathrm{~m}^{3} \mathrm{~s}^{-1}\right)$, relative to the sea floor (Meredith et al., 2011). The ACC plays a pivotal role in setting the global ocean stratification, heat content and overturning circulation (e.g., Gnanadesikan and Hallberg, 2000; Vallis, 2000), and may also set the time scale on which the ocean equilibrates to changes in forcing (Allison et al., 2011; Jones et al., 2011; Samelson, 2011).

9 Moreover, it has been proposed that changes in the strength and latitude of the

*Corresponding author: david.marshall@physics.ox.ac.uk; +44 1865272099 
Southern hemisphere wind jet, due to its impact on the circulation along and across the ACC, may have a profound influence both on past climate variations (e.g., Toggweiler et al., 2006) and anthropogenic climate change in the future (e.g., Fyfe et al., 2007; Le Quéré et al., 2007)

Despite its global climatic importance, there is no consensus on the dynamical processes that set the volume transport of the ACC and its lateral structure, i.e., its meridional excursions with longitude, even in simple models. The traditional, textbook view is that the ACC is driven locally by wind and buoyancy forcing, with geostrophic eddies playing a central role in the equilibrated state (for excellent reviews, see Rintoul et al., 2001; Olbers et al., 2012), although more recent developments have challenged this purely local perspective (e.g., Gnanadesikan and Hallberg, 2000; Fučkar and Vallis, 2007; Mundayet al., 2011). Diagnostic studies with climate models find no clear relation between the volume transport of the ACC and the strength and latitude of the Southern Ocean wind jet (e.g., Russell et al., 2006).

In 1968, Adrian Gill published a seminal paper in which he solved analytically and numerically for the barotropic circulation in an idealized basin with circumpolar connection over a restricted latitude band (Gill, 1968). One of his key objectives was to reconcile zonally-symmetric models of the ACC, in which the volume transport is excessively large, with basin models of the ACC in which the flow consists of a Sverdrup interior and a frictional western boundary current (Stommel, 1957). Key findings were that the volume transport is controlled by the bottom friction and the width of the narrowest constriction in Drake Passage, although the current spreads out to several times this width at other longitudes. However, Gill's model has limited applicability due to its assumption of barotropic dynamics, its excessively large volume transport, and the dependence of the latter on the coefficient of bottom friction.

A key ingredient of contemporary models of the ACC is the intense geostrophic eddy field. In the simplest, zonally-symmetric models, as first developed by Johnson and Bryden (1989), the ACC volume transport is determined through the zonal momentum budget under so called "non-acceleration conditions". Due to the absence of continental barriers at the latitude of Drake Passage, the surface wind stress is mostly balanced by a bottom form stress (Munk and Palmén, 1951) Thus, momentum must be fluxed vertically from the surface to the abyss, which Johnson and Bryden assume is achieved by the eddy form stress. An alternative, but equivalent, physical interpretation is that the equilibrium ACC arises through the competition between the wind-driven Ekman cell (the "Deacon cell") acting to steepen, and the eddy-induced cell generated through baroclinic instability acting to flatten, the isopcynals (e.g., see Danabasoglu et al., 1994). Finally a prediction of the ACC volume transport follows on adopting a closure for the eddy buoyancy fluxes following Green (1970) and Stone (1972), and assuming thermal wind balance and vanishing flow at depth.

However, the ACC is not zonal, but undergoes significant meridional excursions, which are of dynamical importance because the majority of the wind work on the Southern Ocean occurs north of Drake Passage (e.g., see Fig. 14 of Mazloff et al., 2010). Understanding the cause of these meridional excursions is 
important as several studies have suggested that the integral of the wind stress over the circumpolar streamlines of the ACC may serve as a useful predictor of its volume transport (e.g., Ishida, 1994; Allison et al., 2010; LaCasce and Isachsen, 2010). The traditional explanation for these northward excursions is Sverdrup balance (Sverdrup, 1947; Stommel, 1957; LaCasce and Isachsen, 2010). However, if the Ekman driven upwelling is compensated by eddy-induced downwelling, then, at least for that part of the fluid column with circumpolar connection, Sverdrup balance should be modified to include the effect of the eddy-induced downwelling. Intricate interplays between the Sverdrup-like excursions and eddy dynamics are documented in the series of papers by Nadeau and Straub (2009, 2012) and Nadeau and Ferrari (2015).

Recent developments have included the recognition that the ACC cannot be considered independent of the depth of the global pycnocline and the meridional overturning circulation (Gnanadesikan, 1999; Gnanadesikan and Hallberg, 2000). The implication is that the ACC volume transport is influenced not only by Southern Ocean wind forcing and eddies, but also the rate of North Atlantic Deep Water formation (Fučkar and Vallis, 2007), buoyancy forcing (Hogg, 2010) and global diapycnal mixing (Munday et al., 2011).

Finally, it is important to emphasize that the ACC volume transport exhibits far less sensitivity to the surface wind stress in models with explicit, rather than parameterized, eddies, both in equilibrium (Hallberg and Gnanadesikan, 2001; Tansley and Marshall, 2001b; Munday et al., 2013) and during its adjustment (Hallberg and Gnanadesikan, 2006; Hogg and Blundell, 2006; Meredith and Hogg, 2006; Farneti et al., 2010; Farneti and Delworth, 2010). This behavior was first predicted by Straub (1993) on theoretical grounds and has become known as "eddy saturation". Notwithstanding the importance of explicitly resolving eddies, it is important to understand the dynamics of the ACC in models with parameterized eddies, not least because such parameterizations will continue to be used in many climate models for the foreseeable future. Moreover, we have little chance of understanding the dynamics of the ACC with explicit, turbulent eddies if we cannot first understand the dynamics of a quasi-laminar ACC in a model with parameterized eddies.

The goal of this contribution is to develop a simple reduced-gravity model of the AGC that can be used to address three complementary questions:

- How does the volume transport of the ACC vary as the latitude of wind stress forcing is varied?

- Which dynamical processes control the equatorward and poleward excursions of the ACC?

- Can the volume transport of the ACC be predicted from the surface wind stress and model parameters?

The advantage of using a reduced-gravity model is that it is the simplest model that can represent each of the most important elements one might wish to include in a simple theory of the ACC: (i) wind forcing; (ii) basin geometry 
We assume the Rossby

We assume the Rossby number is sufficiently small that inertia can be neglected and the equilibrium momentum equation written:

$$
f \mathbf{k} \times \mathbf{u}+g_{r} \nabla h=\frac{\boldsymbol{\tau}_{s}}{\rho_{0} h}-\frac{r g_{r}}{f} \mathbf{k} \times \nabla h,
$$

where the right-hand side of (2.1) includes a linear drag proportional to the geostrophic velocity. Here $f=f_{0}+\beta y$ is the Coriolis parameter where $f_{0}$ is the Coriolis parameter at the southern boundary and $\beta$ is its meridional gradient, $\mathbf{k}$ is the unit vertical vector, $\mathbf{u}$ is the lateral velocity, $g_{r}$ is the reduced gravity, 


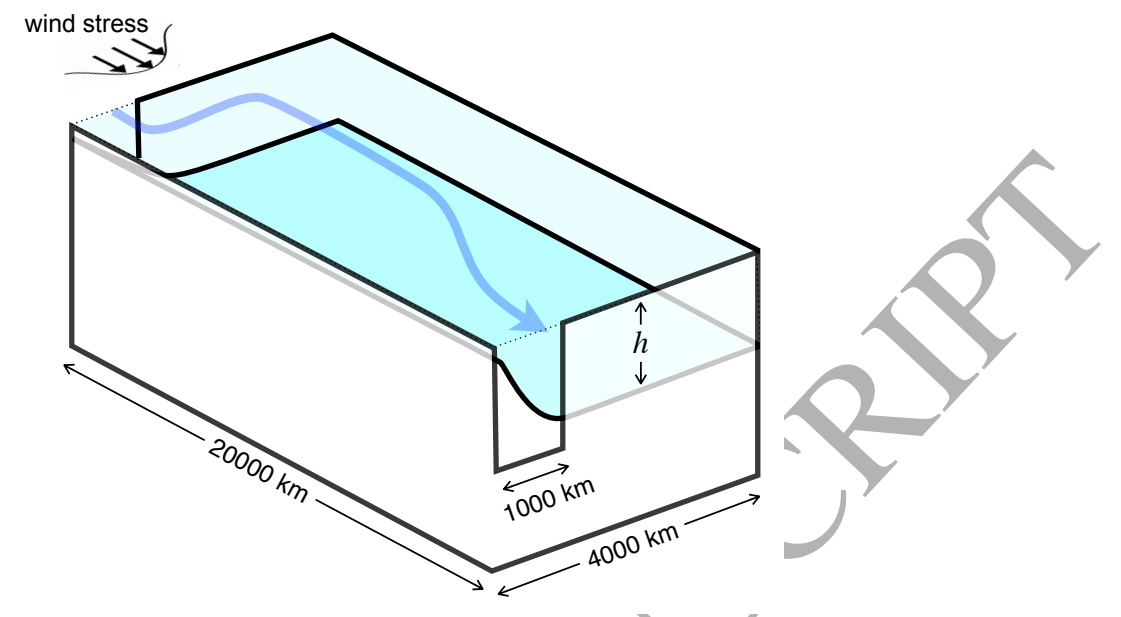

Figure 1: Schematic diagram illustrating the model formulation and domain. Flow is confined to a reduced-gravity layer (shaded) overlaying a motionless abyss. The two layers are separated by a "pycnocline" of depth $h$, across which the density increases abruptly. The upper layer is forced by a prescribed surface wind stress. A re-entrant channel occupies the most southerly quarter of the domain. The model dimensions are indicated on the figure.

$h$ is the layer thickness, $\tau_{s}$ is the surface wind stress, $\rho_{0}$ is a reference density and $r$ is the linear drag coefficient. The linear drag is required to satisfy the nonormal flow boundary condition in the presence of along-shore pressure (layer thickness) variations, the latter being an essential element of the solutions as we shall see in section 4 .

In addition, the flow satisfies a continuity equation,

$$
\nabla \cdot\left(h \mathbf{u}-\kappa_{g m} \nabla h\right)=-\Gamma,
$$

where the second term in (2.2) represents the Gent and McWilliams parameterization of the eddy bolus transport, $\bar{h} \mathbf{u}^{*} \equiv \overline{h^{\prime} \mathbf{u}^{\prime}}=-\kappa_{g m} \nabla h$, where overbars and primes denote time-mean and time-varying components and $\kappa_{g m}$ is the eddy diffusivity (Gent and McWilliams, 1990; Gent et al., 1995). Note that $\bar{h} \mathbf{u}^{*}$ is proportional to the eddy form stress and can therefore also be interpreted as transferting zonal momentum downward from the upper to lower layer (e.g., see the discussions in Hughes, 1997; Olbers, 1998). The term on the right-hand side represent "buoyancy forcing" and is included both to prevent the layer thickness from becoming any smaller than a prescribed minimum value and to impose that the layer outcrops, at the southern boundary, otherwise $\Gamma$ is set to zero;

Boundary conditions on the lateral and northern boundaries are no normal flow and $\kappa_{g m}=0$ to ensure no normal eddy bolus transport. The wind forcing is chosen to be zonal, $\boldsymbol{\tau}_{s}=\tau_{s}(y) \mathbf{i}$, where $\mathbf{i}$ is the unit vector in the $x$ direction; $\tau_{s}$ vanishes along all zonal boundaries such that $\mathbf{n} \cdot \mathbf{k} \times \boldsymbol{\tau}_{s}=0$ where $\mathbf{n}$ is a unit 
where

vector normal to the boundary. With this simplification, the no-normal flow condition can be written as:

$$
\mathbf{n} \cdot\{f \mathbf{k} \times \nabla h-r \nabla h\}=0 .
$$

On the southern boundary we set $h=h_{0}$, where $h_{0}$ is a small value $(10 \mathrm{~m}$ in the solutions shown) as a simple parameterization of buoyancy loss. We also set $h=h_{0}$ on any points where the solution would otherwise give a smaller, value of $h$, i.e., points that might be considered "outcropped"; this is equivalent to introducing an additional volume source at such points through the term $\Lambda$ in $(2.2)$, equivalent to buoyancy gain.

Equations (2.1) and (2.2) combine to give a single elliptic equation for the layer thickness:

$$
-c \frac{\partial h}{\partial x}=\nabla \cdot\left\{\left(\kappa_{g m}+c \delta_{s}\right) \nabla h\right\}-w_{e k}-\mathrm{T}
$$

$$
c(h)=\frac{\beta g_{r} h}{f^{2}}
$$

is the long Rossby wave speed, $\delta_{s}=\gamma / \beta$ is the Stommel boundary layer width ${ }_{172}$ (Stommel, 1948), and $w_{e k}=\mathbf{k} \cdot \nabla \times\left(\boldsymbol{\tau}_{s} / \rho_{0} f\right)$ is the Ekman upwelling velocity 3 (Ekman, 1905).

Equation (2.4) is an advection-diffusion equation containing a westward Rossby advection term (A), a nonlinear diffusion term involving both geostrophic eddy fluxes and linear drag (B), forcing by the Ekman upwelling velocity (C), and buoyancy forcing (D). This advection diffusion equation is proportional to 78 the linear vorticity balance for the upper layer, analogous to the linear vorticity 179 equation in Stommel's models of wind-driven gyres Stommel (1948) and ACC S Stommel (1957). Balances between different combinations of the terms in this equation correspond to different limiting dynamical regimes:

- non-acceleration conditions (terms B and C, neglecting the linear drag by setting $\left.\delta_{s}=0\right)$;

- Sverdrup balance (terms A and C);<smiles>[C]=CC</smiles>

Stommel western boundary current (terms A and B, with the boundary layer width set by eddy diffusion).

Thus, the model seems ideally suited to resolving the relative importance of eddy dynamics, Sverdrup dynamics and western boundary currents in setting 189 the lateral structure and strength of the ACC. 


\subsection{Relation to Gill (1968)}

Equation (2.4) is virtually identical to (2.6) of Gill (1968) for a flat-bottomed, barotropic ocean, except that:

- Gill solves for the barotropic streamfunction in (2.6) whereas we solve for the pycnocline depth, $h$, in (2.4), from which the depth-integrated circulation can be inferred using (2.1);

- (2.4) is nonlinear since the long Rossby speed, $c$, is proportional to the layer thickness;

- the boundary condition (2.3) involves a linear combination of normal and tangential gradients whereas a Dirichlet boundary condition is applied in Gill (1968);

- the inclusion of buoyancy forcing, $\Gamma$, on the right-hand side of (2.4), to allow for outcropping at the southern margin of the domain;

- the linear drag coefficient in Gill is replaced by

$$
r^{*}=r+\frac{\beta \kappa_{g m}}{c},
$$
numerical method are given in the appendix.

where the latter eddy diffusion term dominates in our model;

- $\kappa_{g m}$ is tapered to zero at the boundaries in order to ensure no normal eddy bolus transport.

Thus, the present model might be interpreted as Gill (1968) after a "makeover" to bring it up-to-date with contemporary descriptions of ACC dynamics involving eddy bolus fluxes and the pycnocline depth. Despite the differences between the two models, their solutions have a lot in common.

\section{Model calculations}

Equilibrium solutions to (2.1-2.3) are obtained through a simple relaxation method. The gríd spacing is $50 \mathrm{~km}$ in the basin interior, but decreases in $x$ near the meridional boundaries to enhance resolution within the boundary layers, the finest grid spacing being $0.9 \mathrm{~km}$ adjacent to the boundaries. Details of the The wind stress has the same generic spatial profile:

$$
\tau_{s}^{(x)}= \begin{cases}\tau_{0} \sin ^{2}\left(\pi \frac{y-y_{s}}{y_{n}-y_{s}}\right) & \text { if } y_{s} \leq y \leq y_{n} \\ 0 & \text { otherwise. }\end{cases}
$$

However, the latitudinal extent and strength of the wind is varied through the parameters $\tau_{0}, y_{s}$ and $y_{n}$, as summarized in Table 1 . The calculations are clustered into sets in which the wind stress profile has widths of $y_{0} / 4, y_{0} / 2,3 y_{0} / 4$ 
$255 y_{n}=y_{0}$ in (3.1) with $\tau_{0}=0.2 \mathrm{~N} \mathrm{~m}^{-2}$.

Plotted in Fig. 2 are: the layer thickness, $h$; the transport streamfunction, $\psi$; the Ekman upwelling, $w_{e k}$; the eddy-induced upwelling,

$$
w_{e d d y}=-\nabla \cdot\left(\kappa_{g m} \nabla h\right) ;
$$

258 259

and $y_{0}$ (recall that the Drake Passage width is $y_{0} / 4$ and the overall basin width $\left.y_{0}\right)$. The wind stress profiles are, in turn, shifted northward across different starting latitudes as space allows, and four different wind strengths are employed. While the largest wind stress of $0.4 \mathrm{~N} \mathrm{~m}^{-2}$ is stronger than in reality, of the wind profiles are unrealistically narrow in order to isolate the effect quired to maintain a realistic overall momentum input and circumpolar volume transport. The calculations are labelled "Wsnc" where $s$ and $n$ are $y_{s}$ and $y_{n}$ in $10^{3} \mathrm{~km}$ from the southern boundary, and $\tau$ indicates the maximum wind stress in $0.1 \mathrm{~N} \mathrm{~m}^{-2}$ (except 0 is used for $\tau=0.05 \mathrm{~N} \mathrm{~m}^{-2}$ ).

In each experiment, the layer thickness is pinned to $h_{0}=10 \mathrm{~m}$ on the southern boundary, representing the effect of buoyancy loss in outcropping the pycnocline. In most cases, the Gent and McWilliams eddy diffusivity and linear drag coefficients have control values of $1000 \mathrm{~m}^{2} \mathrm{~s}^{-1}$ and $10^{-7} \mathrm{~s}^{-1}$ respectively, except that the eddy diffusivity is scaled to zero adjacent to the western, eastern and northern boundaries over the Stommel boundary layer scale,

$$
\kappa_{g m}=\kappa_{g m 0}\left(1-e^{\beta x / r}\right), \quad \text { etc, }
$$

in order to ensure no normal eddy bolus transport. This choice is fairly ad-hoc, but does not appear critical as long as the tapering scale is significantly shorter than the overall width of the boundary layer, $\kappa_{g m} / c$ (see section 4.3). Ensuring that these nested boundary layers are well resolved is the primary motivation for enhancing zonal resolution adjacent to the western (and eastern) boundaries. Four additional calculations are reported in which the Gent and McWilliams eddy diffusivity is increased and decreased by a factor of 2 , and the linear drag eefficient is increased by factors of 3 and 10 , about the calculation W022; these

The equilibrium layer thickness at the northern edge of Drake Passage and the Drake Passage volume transport are listed in the final two columns of Table 1. In the following sections, we discuss the lateral structure of a typical solution, sensitivity of the solution to the latitude of the wind jet, and the relation of the Drake Passage volume transport to the surface wind stress.

\section{Lateral structure of a typical solution}

In this section, we discuss the lateral structure of a typical solution, W042, through/reference to the diffusive Rossby wave equation (2.4). As indicated in Table 1, the solution has a fairly realistic, basin-wide wind forcing: $y_{s}=0$,

the sum of the "geostrophic" upwelling (equivalent to planetary vorticity advection),

$$
w_{\text {geos }}=-c \partial h / \partial x,
$$




\begin{tabular}{|c|c|c|c|c|c|c|}
\hline name & $\begin{array}{ll}\text { wind } & \text { latitude } \\
(\mathrm{km}) & \\
\end{array}$ & $\begin{array}{l}\tau_{0} \\
\left(N m^{-2}\right)\end{array}$ & $\begin{array}{l}\kappa_{G M 0} \\
\left(m^{2} s^{-1}\right)\end{array}$ & $r\left(s^{-1}\right)$ & $h_{d p}(\mathrm{~m})$ & $T_{d p}(\mathrm{~Sv})$ \\
\hline W010 & $0-1000$ & 0.05 & 1000 & $1 \times 10^{-7}$ & 229 & 2 \\
\hline W011 & $0-1000$ & 0.1 & 1000 & $1 \times 10^{-7}$ & 444 & 9 \\
\hline W012 & $0-1000$ & 0.2 & 1000 & $1 \times 10^{-7}$ & 864 & 35 \\
\hline W014 & $0-1000$ & 0.4 & 1000 & $1 \times 10^{-7}$ & 1662 & 128 \\
\hline W120 & $1000-2000$ & 0.05 & 1000 & $1 \times 10^{-7}$ & 214 & 2 \\
\hline W121 & $1000-2000$ & 0.1 & 1000 & $1 \times 10^{-7}$ & 415 & 9 \\
\hline W122 & $1000-2000$ & 0.2 & 1000 & $1 \times 10^{-7}$ & 778 & 31 \\
\hline W124 & $1000-2000$ & 0.4 & 1000 & $1 \times 10^{-7}$ & 1406 & 101 \\
\hline W230 & $2000-3000$ & 0.05 & 1000 & $1 \times 10^{-7}$ & 231 & 3 \\
\hline W231 & $2000-3000$ & 0.1 & 1000 & $1 \times 10^{-7}$ & 414 & 9 \\
\hline W232 & $2000-3000$ & 0.2 & 1000 & $1 \times 10^{-7}$ & 713 & 27 \\
\hline W234 & $2000-3000$ & 0.4 & 1000 & $1 \times 10^{-7}$ & 1179 & 72 \\
\hline W340 & $3000-4000$ & 0.05 & 1000 & $1 \times 10^{-7}$ & 300 & 5 \\
\hline W341 & $3000-4000$ & 0.1 & 1000 & $1 \times 10^{-7}$ & 479 & 13 \\
\hline W342 & $3000-4000$ & 0.2 & 1000 & $1 \times 10^{-7}$ & 750 & 30 \\
\hline W344 & $3000-4000$ & 0.4 & 1000 & $1 \times 10^{-7}$ & 1194 & 75 \\
\hline W020 & $0-2000$ & 0.05 & 1000 & $1 \times 10^{-7}$ & 423 & 9 \\
\hline W021 & $0-2000$ & 0.1 & 1000 & $1 \times 10^{-7}$ & 806 & 33 \\
\hline W022 & $0-2000$ & 0.2 & 1000 & $1 \times 10^{-7}$ & 1507 & 114 \\
\hline W024 & $0-2000$ & 0.4 & 1000 & $1 \times 10^{-7}$ & 2743 & 375 \\
\hline W130 & $1000-3000$ & 0.05 & 1000 & $1 \times 10^{-7}$ & 381 & 8 \\
\hline W131 & $1000-3000$ & 0.1 & 1000 & $1 \times 10^{-7}$ & 671 & 24 \\
\hline W132 & $1000-3000$ & 0.2 & 1000 & $1 \times 10^{-7}$ & 1136 & 67 \\
\hline W134 & $1000-3000$ & 0.4 & 1000 & $1 \times 10^{-7}$ & 1852 & 176 \\
\hline W240 & $2000-4000$ & 0.05 & 1000 & $1 \times 10^{-7}$ & 388 & 8 \\
\hline W241 & $2000-4000$ & 0.1 & 1000 & $1 \times 10^{-7}$ & 622 & 21 \\
\hline W242 & $2000-4000$ & 0.2 & 1000 & $1 \times 10^{-7}$ & 970 & 50 \\
\hline W244 & $2000-4000$ & 0.4 & 1000 & $1 \times 10^{-7}$ & 1477 & 114 \\
\hline W030 & $0-3000$ & 0.05 & 1000 & $1 \times 10^{-7}$ & 533 & 15 \\
\hline W031 & $0-3000$ & 0.1 & 1000 & $1 \times 10^{-7}$ & 947 & 46 \\
\hline W032 & $0-3000$ & 0.2 & 1000 & $1 \times 10^{-7}$ & 1634 & 136 \\
\hline W034 & $0-3000$ & 0.4 & 1000 & $1 \times 10^{-7}$ & 2748 & 383 \\
\hline W140 & $1000-4000$ & 0.05 & 1000 & $1 \times 10^{-7}$ & 472 & 12 \\
\hline W141 & $1000-4000$ & 0.1 & 1000 & $1 \times 10^{-7}$ & 770 & 31 \\
\hline W142 & $1000-4000$ & 0.2 & 1000 & $1 \times 10^{-7}$ & 1219 & 78 \\
\hline W144 & $1000-4000$ & 0.4 & 1000 & $1 \times 10^{-7}$ & 1877 & 182 \\
\hline W040 & $0-4000$ & 0.05 & 1000 & $1 \times 10^{-7}$ & 578 & 18 \\
\hline W041 & $0-4000$ & 0.1 & 1000 & $1 \times 10^{-7}$ & 964 & 48 \\
\hline W042 & $0-4000$ & 0.2 & 1000 & $1 \times 10^{-7}$ & 1568 & 127 \\
\hline W044 & $0-4000$ & 0.4 & 1000 & $1 \times 10^{-7}$ & 2501 & 320 \\
\hline W022K- & $0-2000$ & 0.2 & 500 & $1 \times 10^{-7}$ & 2425 & 292 \\
\hline W022K+ & $0-2000$ & 0.2 & 2000 & $1 \times 10^{-7}$ & 836 & 35 \\
\hline W022R+ & $0-2000$ & 0.2 & 1000 & $3 \times 10^{-7}$ & 1386 & 95 \\
\hline $\mathrm{W} 022 \mathrm{R}++$ & $0-2000$ & 0.2 & 1000 & $10 \times 10^{-7}$ & 1112 & 61 \\
\hline
\end{tabular}

Table 1: Summary of the model calculations. The final two columns show equilibrium values of the layer thickness at the northern tip of, and the volume transport through, the model Drake Passage. 
(a) layer thickness (Cl: $200 \mathrm{~m})$

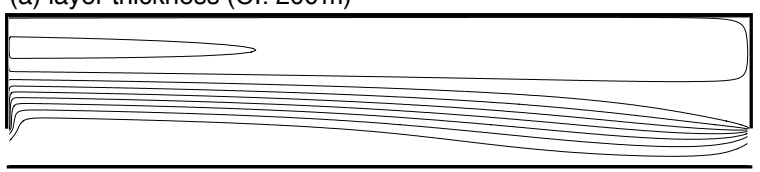

(b) streamfunction (Cl: 20Sv)

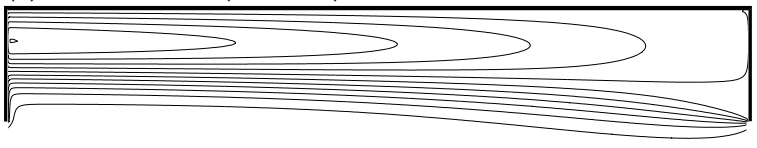

(c) Ekman upwelling $\left(10^{-6} \mathrm{~ms}^{-1}\right)$
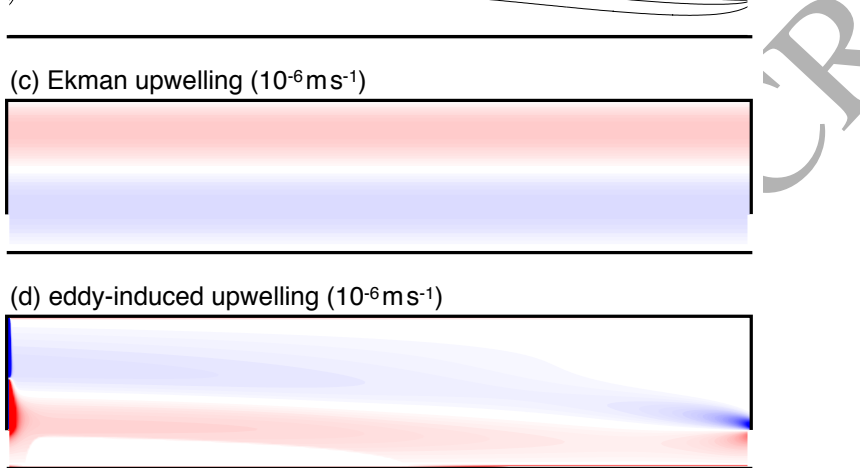

(e) geostrophic + frictional upwelling $\left(10^{-6} \mathrm{~ms}^{-1}\right)$

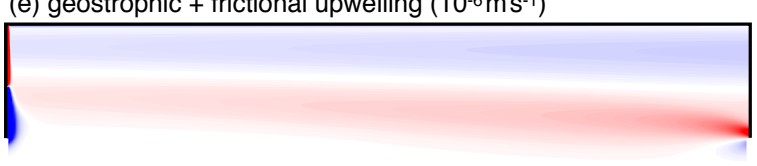

(f) "buoyancy forcing" $\left(10^{-6} \mathrm{~ms}^{-1}\right)$

Figure 2: Lateral structure of solution W042. Plotted are: (a) the layer thickness, $h$ (contour interval $200 \mathrm{~m}$, increasing northward from $h_{0}=10 \mathrm{~m}$ on the southern boundary); (b) the transport streamfunction, $\psi$, as defined in (4.5) (contour interval $20 \mathrm{~Sv}$ ); (c) the Ekman upwelling, $w_{e k}$; (d) the eddy-induced upwelling, $w_{e d d y}$, as defined in $(4.1)$; (e) the sum of the geostrophic and frictional upwellings, $w_{\text {geos }}+w_{f r i c}$, as defined in (4.2) and (4.3); (f) "buoyancy forcing" $\Gamma$. Panels (c) - (f) are shaded in units of $10^{-6} \mathrm{~m} \mathrm{~s}^{-1}$. Red shading corresponds to negative values, i.e., processes that deepen the pycnocline or warm the ocean; blue shading corresponds to negative values, i.e., processes that shallow the pycnocline or cool the ocean.

260 and "frictional" upwelling (equivalent to the frictional vorticity sink),

$$
w_{\text {fric }}=-\nabla \cdot\left(c \delta_{s} \nabla h\right) ;
$$


and the "buoyancy forcing", $\Gamma$. The latter five quantities correspond to the terms in the elliptic equation (2.4), defined such that

$$
w_{e k}+w_{\text {eddy }}+w_{\text {geos }}+w_{\text {fric }}+\Gamma=0 .
$$

Red shading corresponds to negative values, i.e., processes that deepen the pycnocline or warm the ocean; blue shading corresponds to negative values, i. processes that shallow the pycnocline or cool the ocean. The geostrophic and frictional upwellings are combined because they are calculated jointly in the numerical code (see Appendix).

The transport streamfunction is defined such that

$$
h \mathbf{u}-\kappa_{g m} \nabla h=\mathbf{k} \times \nabla \psi
$$

where $h \mathbf{u}$ includes the Ekman transport velocity and it is implicitly assumed that buoyancy forcing is negligible. In practice, buoyancy forcing mostly occurs south of the model ACC jet and thus we calculate $\psi$ by integrating the zonal component of (4.5) southward from the northern boundary; this means that streamfunction values south of any buoyancy forcing are degenerate. (We did consider first decomposing the depth-integrated transport into rotational and divergent components. However, in practice the latter component is small and has little discernible effect on the structure of any of the streamfunction fields plotted in this manuscript.)

The solution consists of a circumpolar current with a volume transport of $127 \mathrm{~Sv}$ and a subtropical "supergyre" (cf. Ridgway and Dunn, 2007) with a volume transport of $101 \mathrm{~Sv}$. The layer thickness has its minimum allowed value of $10 \mathrm{~m}$ at the southern boundary and in an outcropped region in the southwest of the domain. The latter is most easily identified by the positive "buoyancy forcing" in Fig. 2(f). The layer thickness has a value of $1568 \mathrm{~m}$ at the northern tip of the model Drake Passage, and reaches a maximum of $1843 \mathrm{~m}$ at the center of the supergyre.

Physically, it is easiest to interpret the solution for the circumpolar current by tracing its path backwards from the model Drake Passage. The following discussion closely follows that of Gill (1968) (sections 3-5); the reader is also referred to de Ruijter (1980) for further discussion.

\subsection{Diffusive jet regime}

First we start with the flow immediately upstream of Drake Passage. An expanded view of the eddy upwelling, geostrophic plus frictional upwelling and layer thickness contours is shown in Fig. 3. In this region, the elliptic equation (2.4) is well approximated by

$$
-c \frac{\partial h}{\partial x} \approx \frac{\partial}{\partial y}\left(\kappa_{g m} \frac{\partial h}{\partial y}\right) .
$$

where the left-hand side of (4.6) is the Lagrangian rate of change of $h$ following a Rossby wave trajectory. Treating $\kappa_{g m}$ and $c$ as a constant (in practice $c$ is a 


\subsection{Basin interior}

Away from the boundaries, the circumpolar current satisfies an approximate three-way balance in (2.4) involving Ekman upwelling, and eddy-induced and geostrophic downwelling (Fig. 2). This is equivalent to a modified Sverdrup balance,

$$
\beta v h \approx f\left(w_{e k}+w_{e d d y}\right),
$$

linear function of $h),(4.6)$ can be differentiated with respect to $y$ to give the same equation for the gradient,

$$
-c \frac{\partial}{\partial x}\left(\frac{\partial h}{\partial y}\right) \approx \kappa_{g m} \frac{\partial^{2}}{\partial y^{2}}\left(\frac{\partial h}{\partial y}\right) ;
$$

this is the standard equation for diffusion of a tracer from an initial point source.

$$
\frac{\partial h}{\partial y} \propto \sqrt{\frac{c}{4 \pi \kappa_{g m}\left(x_{0}-x\right)}} \exp \left\{\frac{c\left(y-y_{d p}\right)^{2}}{4 \pi \kappa_{g m}\left(x_{0}-x\right)}\right\},
$$

where $y_{d p}=y_{0} / 4$ is the value of $y$ at the northern tip of Drake Passage. Finally, (4.8) can be integrated across the jet to give the solution for the layer thickness,

$$
h \approx h_{0}+\frac{\Delta h}{2}\left(1+\operatorname{erf}\left\{\sqrt{\frac{c}{4 \pi \kappa_{g m}\left(x_{0}-x\right)}}\left(y-y_{d p}\right)\right\}\right),
$$

here $\operatorname{erf}\{\cdots\}$ is the error function (e.g., page 297, Gautschi, 1964).

Taking the argument of the error function to be \pm 1 allows us to estimate width of the jet, $\Delta y$, as the spacing between the contours $h=h_{0}+0.08 \Delta h$,

$$
\Delta y \approx 4 \sqrt{\frac{\kappa_{g m}\left(x_{0}-x\right)}{c}} .
$$

Putting in typical values of $c \approx 2 \times 10^{-2} \mathrm{~ms}^{-1}$, appropriate with $h \approx 10^{3} \mathrm{~m}$ $=10^{-4} \mathrm{~s}^{-1}$ at the latitude of the model Drake Passage, $\kappa_{g m}=10^{3} \mathrm{~m}^{2} \mathrm{~s}^{-1}$ broadening of the jet upstream of the model Drake Passage in Fig. 2(a). At a more detailed level, it is also evident that the jet broadens over a shorter distance upstream of the model Drake Passage to the south, consistent with the

Broadening of the ACC upstream of Drake Passage is realistic (see, e.g., the sservation-constrained Southern Ocean State Estimate of Mazloff et al., 2010), vivestigation, not least because Rossby wave propagation is eastward rather than westward, in the ACC due to Doppler-shifting by the depth-mean flow (Klocker and Marshall, 2014).

including the compensating effect of eddies on the net upwelling. Thus, the solution shows elements of both the first and second limiting paradigms discussed 
(a) eddy-induced upwelling $\left(10^{-6} \mathrm{~m} \mathrm{~s}^{-1}\right)$

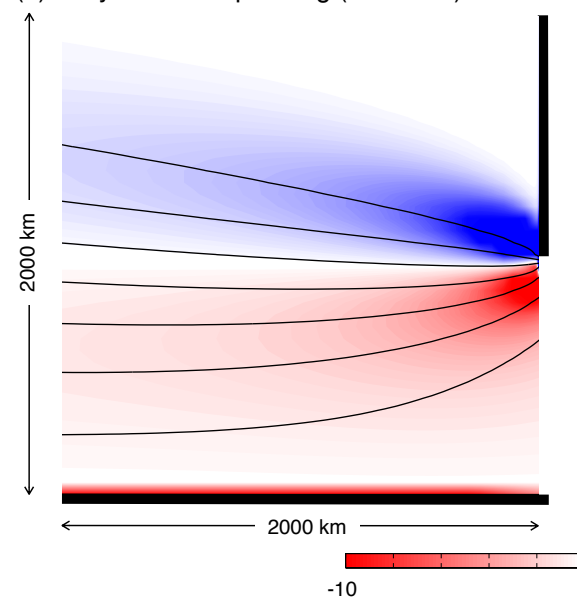

(b) geostrophic + frictional upwelling $\left(10^{-6} \mathrm{~ms}^{-1}\right)$

Figure 3: Expanded view of the dynamical balance in the region immediately upstream of the model Drake Passage in solution W042. Shading shows (a) the eddy-induced upwelling, and (b) the sum of the geostrophic and frictional upwellings, $w_{\text {geos }}+w_{\text {fric }}\left(\right.$ units: $10^{-6} \mathrm{~m} \mathrm{~s}^{-1}$ ). Superimposed on each are layer thickness contours with a contour interval of $200 \mathrm{~m}$ (the southernmost contour being the $200 \mathrm{~m}$ contour). with the width of the boundary current set by the Gent and McWilliams eddy diffusivity,

$$
\delta_{w b c} \sim \frac{c}{\kappa_{g m}} .
$$

Taking $c \approx 2 \times 10^{-2} \mathrm{~m} \mathrm{~s}^{-1}$ and $\kappa_{g m}=10^{3} \mathrm{~m}^{2} \mathrm{~s}^{-1}$ gives $\delta_{w b c} \sim 50 \mathrm{~km}$. In contrast, the Stommel boundary layer width, $\delta_{s}=r / \beta=5 \mathrm{~km}$, an order of 340 magnitude smaller. A similar result has been previously obtained and discussed 341 by Eden and Olbers (2010). 
(a) eddy-induced upwelling $\left(10^{-6} \mathrm{~ms}^{-1}\right)$

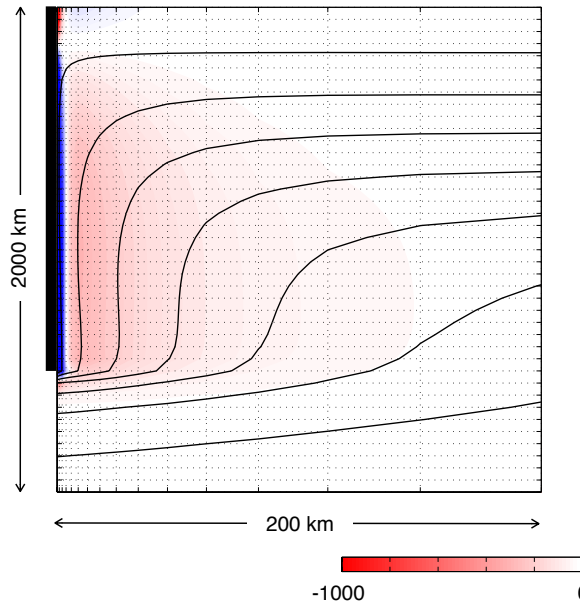

(b) geostrophic + frictional upwelling $\left(10^{-6} \mathrm{~m} \mathrm{~s}^{-1}\right)$

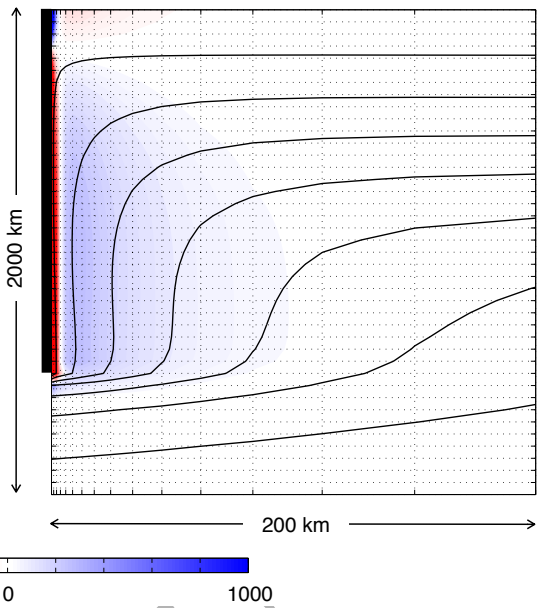

Figure 4: Expanded view of the dynamical balance in the western boundary current of the circumpolar current in solution W042. Note that the zonal dimension is expanded by a factor 10 more than the meridional dimension. Shading shows (a) the eddy-induced upwelling, and (b) the sum of the geostrophic and frictional upwellings, $w_{\text {geos }}+w_{\text {fric }}$ (units: $10^{-6} \mathrm{~m} \mathrm{~s}^{-1}$; note that the color scale is for values 100 times larger than in previous figures). Superimposed on each are layer thickness contours with a contour interval of $200 \mathrm{~m}$ (the southernmost contour being the $200 \mathrm{~m}$ contour). Also indicated by tick marks and dotted lines is the model grid, indicating the increase in resolution in the zonal direction towards the boundary.

55

An expanded view of the eddy upwelling, geostrophic plus frictional upwelling and layer thickness contours is shown in Fig. 4; the zonal scale is magnified by an order of magnitude relative to the meridional scale in order to reveal the balances adjacent to the solid boundary. The western boundary current width is in accord with the approximate balance (4.12), within which there is eddy-induced downwelling due to the convergence of the eddy thickness fluxes which decay with $x$. However, there is a narrow region adjacent to the boundary, of width set by the Stommel boundary layer thickness, in which there is intense eddy-induced upwelling due to the imposed vanishing of the eddy bolus transport at the solid boundary. However, the details of the width of this layer of eddy-induced upwelling appear to be of secondary importance to the overall structure of the solution, as long as it is a small fraction of the overall width of the boundary current (not shown).

\subsection{Outcropping and the residual circulation}

There is a region in the southwestern corner of Fig 2(f) in which the net upwelling does not vanish. This corresponds to a region in which the layer is outcropped, analogous to the separated region in Fig. 3(d) of Gill (1968). In this outcropped region, the eddy-induced, geostrophic and frictional upwellings all vanish and hence the Ekman upwelling can only be balanced by positive 
(a)

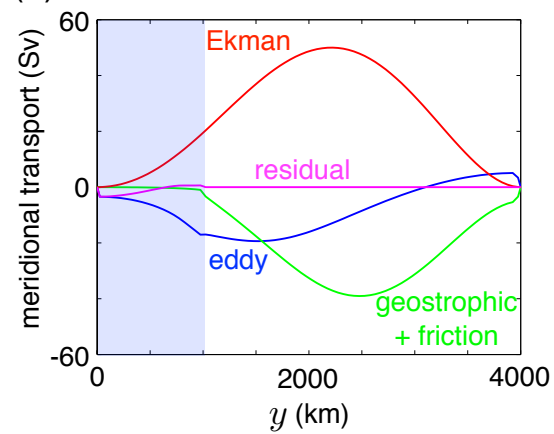

(b)

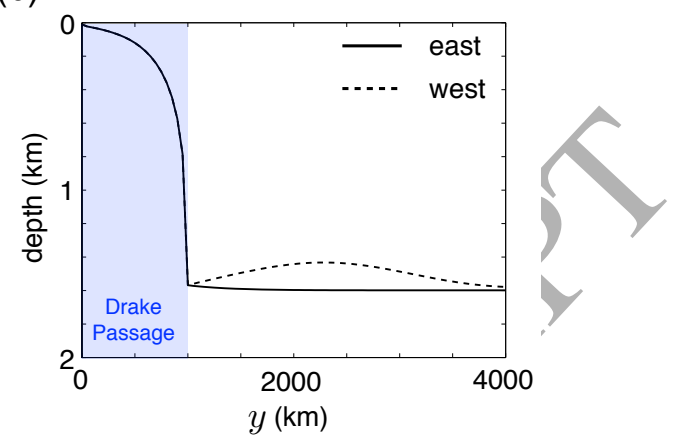

Figure 5: (a) Net northward volume transports in solution W042, decomposed into Ekman (red), eddy (blue), and geostrophic plus frictional components (green); also shown is the residual volume transport (magenta). (b) Variation of the layer thickness, $h$, with meridional distance, $y$, along the eastern (solid lines) and western (dashed lines) boundaries in solution W042; within the model Drake Passage, the eastern and western values are equal by definition.

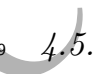

Finally we briefly discuss the zonal momentum budget, equivalent to the meridional volume transport budget shown in Fig. 5(a), after rearranging the zonal component of the momentum equation (2.1) for $v$, multiplying by $h$ and integrating zonally (also see Olbers, 1998).

Over the circumpolar latitudes, the Ekman and eddy-induced meridional 35 volume transports balance to leading order, equivalent to a balance between the 
surface wind stress and eddy form stress as assumed by Johnson and Bryden (1989). The slight imbalance is due to the residual southward volume transport across the circumpolar current, already discussed in section 4.4, and an even smaller contribution from friction.

North of the model Drake Passage, the eddy-induced volume transport continues to compensate for the Ekman transport, but there is an increasingly large contribution from the geostrophic transport, equivalent to an east-west pressure gradient. The origin of this pressure gradient is clear from Fig. 5(b) in which the layer thickness is shown along $x=0$ and $x=x_{0}$, corresponding to the western and eastern boundaries. Within the circumpolar latitudes, the western and eastern layer thicknesses are equal, by definition, but further north the layer thickness, and hence pressure, drops along the western boundary, whereas it is nearly constant along the eastern boundary. This basin-wide zonal pressure has an impact on the volume transport of the circumpolar current, as we shall discuss further in section 6 .

\section{Sensitivity to latitude of the wind jet}

In this section, we focus on the sensitivity of the solution to the latitude of the wind jet by discussing the four solutions W014, W124, W234 and W344 (Figs 6-9). While the wind jet is unrealistically narrow in these solutions, this has the advantage of isolating the effect of wind forcing in different parts of the basin and, in particular, the role played by Sverdrup balance in setting the lateral structure and strength of the cireumpolar current. The magnitude of the maximum wind stress is relatively large in each of these experiments, at $0.4 \mathrm{~N} \mathrm{~m}^{-2}$, but we justify this value by noting that momentum is transferred to the ocean over a restricted latitude band; for example, the net momentum input is half that in the solution W022 discussed in section 4 .

\subsection{Lateral structure}

In the first solution, W014 (Fig. 6), the wind forcing is confined to the latitude band of the model Drake Passage. The solution corresponds almost exactly to the first paradigm discussed in section 1 due to Johnson and Bryden (1989), with pointwise compensation between the Ekman and eddy-induced upwellings. The slight imbalance is due to the frictional upwelling (indeed, if the eddy transfer coefficient is reinterpreted as $\kappa_{g m}+c \delta_{s}$, then the compensation is virtually exact). Note that there is no outcropping of the layer except on the southern boundary where this is imposed through the boundary condition.

In the second solution, W124 (Fig. 7), the wind forcing is entirely to the north of the model Drake Passage. Nevertheless, the dominant balance over most of the basin remains between the Ekman and eddy-induced upwellings, with weaker contribution from the geostrophic upwelling (and to a lesser extent, the frictional upwelling). The slight reduction in the eddy-induced upwelling compared with solution W014 (evident from the larger contribution from geostrophic upwelling in Fig. 7(e)) means that the layer interface slopes 
(a) layer thickness (Cl: $200 \mathrm{~m})$

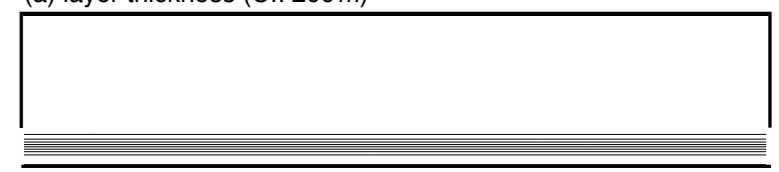

(b) streamfunction (Cl: 20Sv)

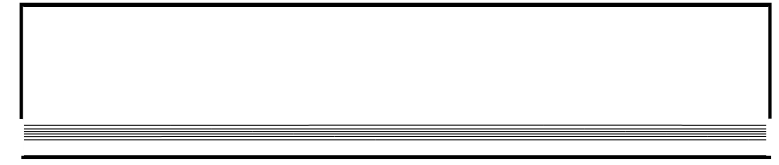

(c) Ekman upwelling $\left(10^{-6} \mathrm{~ms}^{-1}\right)$

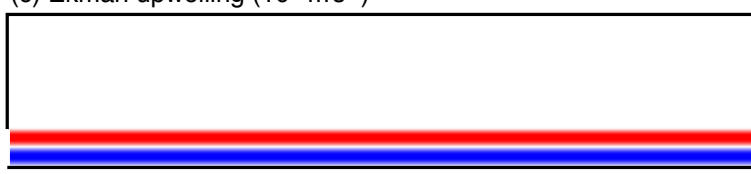

(d) eddy-induced upwelling $\left(10^{-6} \mathrm{~ms}^{-1}\right)$

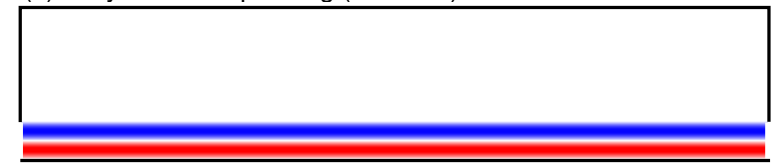

(e) geostrophic + frictional upwelling $\left(10^{-6} \mathrm{~ms}^{-1}\right)$

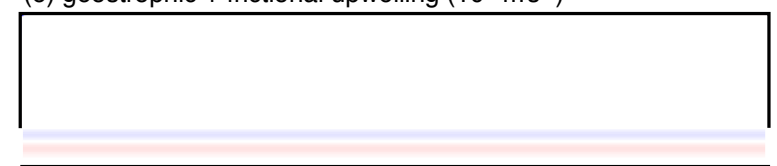

(f) "buoyancy forcing" $\left(10^{-6} \mathrm{~ms}^{-1}\right)$

Figure 6: Lateral structure of solution W014. The plotted fields are as in Fig. 2.

${ }_{428}$ less steeply in solution W124, leading to a slightly weaker circumpolar volume transport through thermal wind balance. Note also that the layer outcrops over a narrow band at the southernmost tip of the prescribed Ekman upwelling 431 (south of this strip, the layer is close to, but not quite, outcropped). As dis432 cussed in section 4.4, this outcropping is required in order to balance the net 433 eddy-induced upwelling at the southern boundary.

However, the most important result of the second and remaining solutions ${ }_{435}$ is that Sverdrup balance does not set the volume transport through Drake Pas- 
(a) layer thickness $(\mathrm{Cl}: 200 \mathrm{~m})$

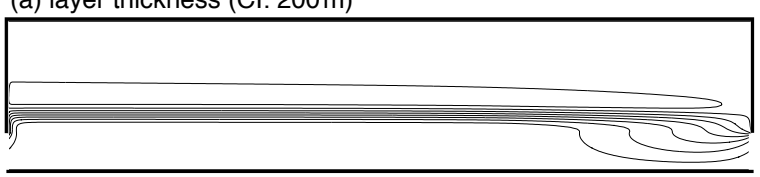

(b) streamfunction (Cl: 20Sv)

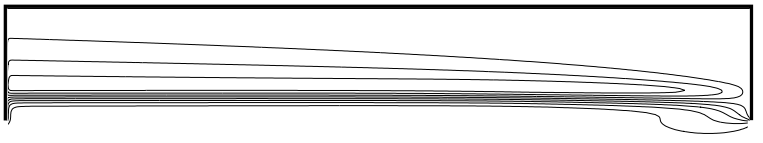

(c) Ekman upwelling $\left(10^{-6} \mathrm{~ms}^{-1}\right)$

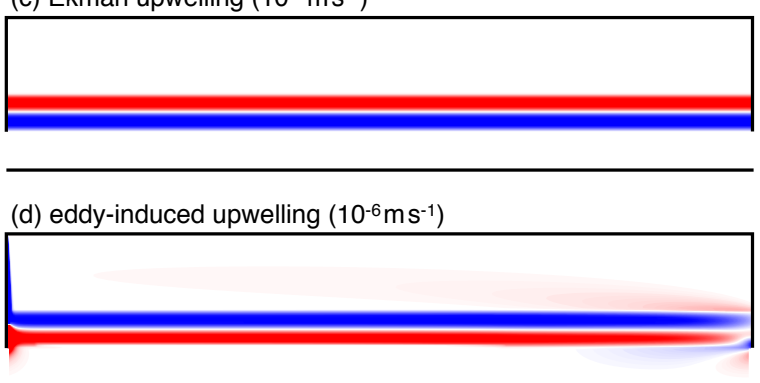

(e) geostrophic + frictional upwelling $\left(10^{-6} \mathrm{~ms}^{-1}\right)$

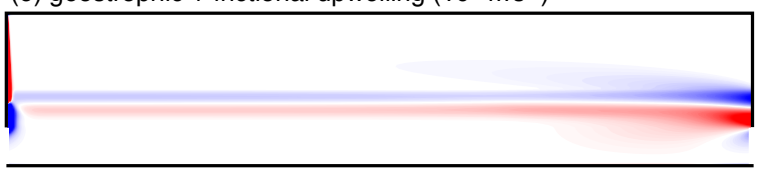

(f) "buoyancy forcing" $\left(10^{-6} \mathrm{~ms}^{-1}\right)$
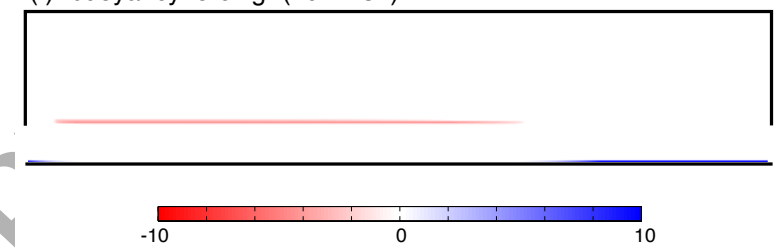

Figure 7: Lateral structure of solution W124. The plotted fields are as in Fig. 2.

436 sage as proposed by Stommel (1957). In solution W124, there is no Ekman upwelling at the latitude of the northern tip of Drake Passage and thus Stommel's paradigm predicts no circumpolar transport, inconsistent with the plotted solution. Instead, the poleward migration of the fluid columns is achieved through an "eddy Sverdrup balance" between the eddy-induced and geostrophic upwellings, or equivalently

$$
\beta v h \approx f w_{e d d y}
$$


(a) layer thickness (Cl: $200 \mathrm{~m})$

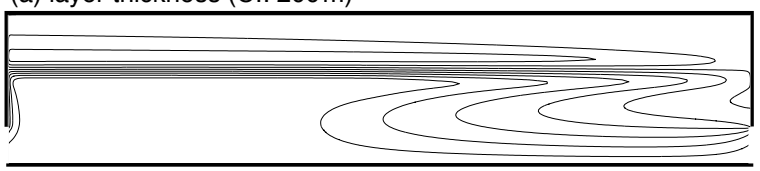

(b) streamfunction (Cl: 20Sv)

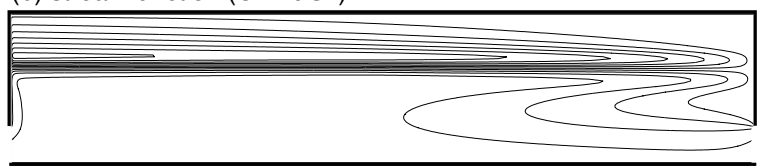

(c) Ekman upwelling $\left(10^{-6} \mathrm{~ms}^{-1}\right)$

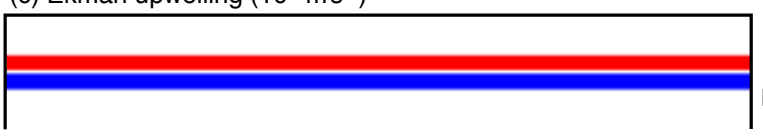

(d) eddy-induced upwelling $\left(10^{-6} \mathrm{~ms}^{-1}\right)$

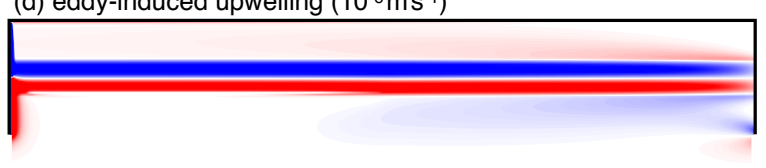

(e) geostrophic + frictional upwelling $\left(10^{-6} \mathrm{~ms}^{-1}\right)$

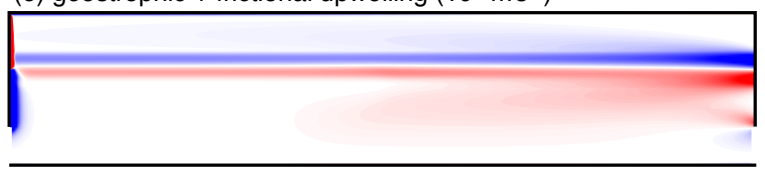

(f) "buoyancy forcing" (10-6 $\left.\mathrm{ms}^{-1}\right)$
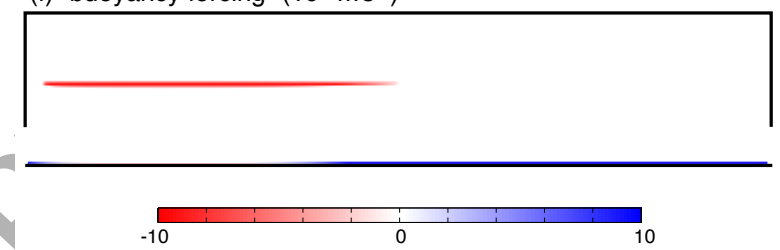

Figure 8: Lateral structure of solution W234. The plotted fields are as in Fig. 2.

${ }_{442}$ In the third and fourth solutions, W234 (Fig. 8) and W344 (Fig. 9), in ${ }_{443}$ which the wind forcing is shifted even further north, a similar regime to the second solution persists, except that the southward deflection of the circumpolar 445 current occurs over a larger area and there is some retroflection of the current. 446 In each case, the narrow outcropped region migrates northward with the wind 447 jet. For the most northerly wind profile, the retroflection fills the zonal width 448 of the basin and there is a weak quasi-zonal transport within the circumpolar 449 latitudes. 


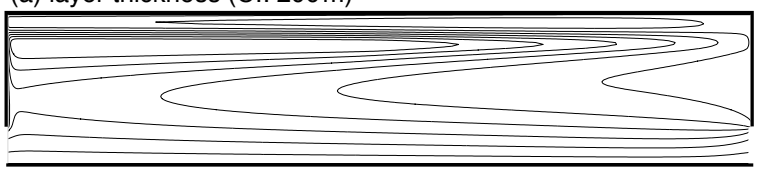

(b) streamfunction (Cl: 20Sv)

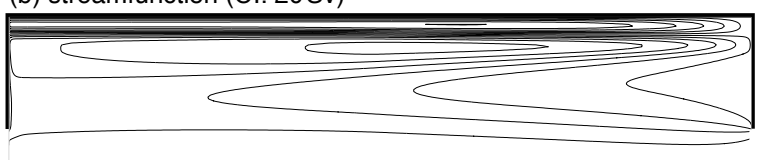

(c) Ekman upwelling $\left(10^{-6} \mathrm{~ms}^{-1}\right)$

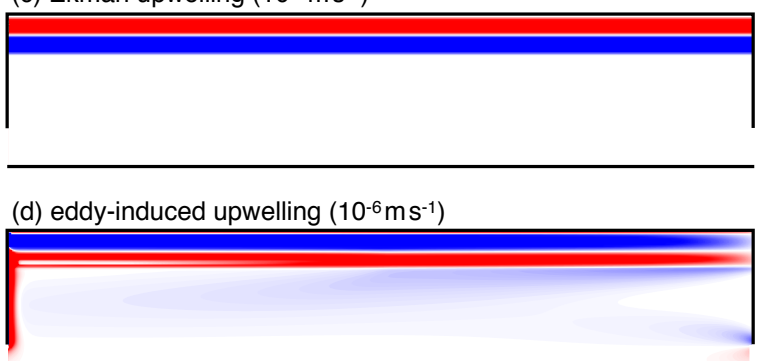

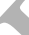

(e) geostrophic + frictional upwelling $\left(10^{-6} \mathrm{~ms}^{-1}\right)$

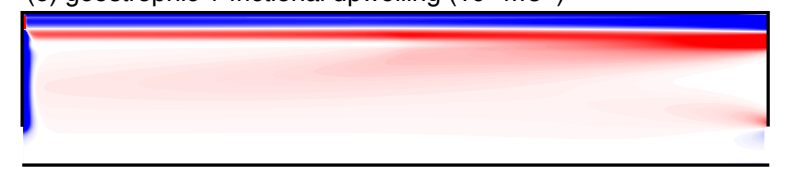

(f) "buoyancy forcing" $\left(10^{-6} \mathrm{~ms}^{-1}\right)$

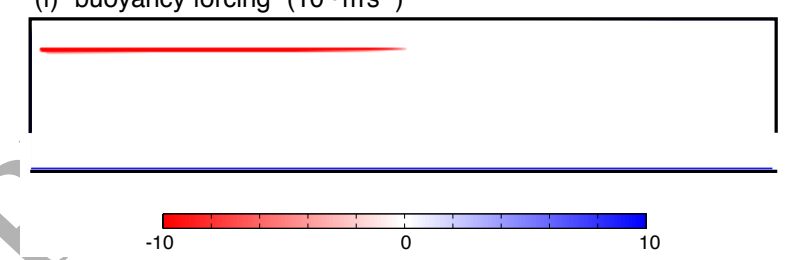

Figure 9: Lateral structure of solution W344. The plotted fields are as in Fig. 2.

450 5.2. Zonal momentum budget

The net meridional volume transport, equivalent to the zonal momentum budget (see section 4.5), and the layer thickness profiles along the eastern and western boundaries, are plotted in Fig. 10 for each of the four solutions.

Consistent with the preceding discussion, the Ekman and eddy-induced up45 wellings compensate, aside from a small frictional contribution, when the wind 456 forcing is confined to the latitudes of the model Drake Passage (panel (a)) and 457 there is no residual volume transport. Thus, the momentum balance is be- 
tween the surface wind stress and eddy form stress, consistent with Johnson and Bryden (1989).

However, as the wind jet is shifted progressively further north (panels (b)(d)), the geostrophic contribution to the meridional volume transport becomes increasingly important. This is associated with an increasingly large zonal pressure gradient from the shallowing of the layer interface along the western boundary. Nevertheless, note that the eddy contribution remains substantial in each of the four solutions. In addition, the magnitude of the residual southward volume transport increases as the wind profile shifts north. The reason for this remains unclear, but it may be related to the scaling of the Ekman upwelling in the outcropped region with the inverse Coriolis parameter.

\subsection{Drake Passage volume transport}

Finally in Fig. 11 we plot the Drake Passage volume transport for each of the four solutions against the latitude of the peak wind stress, and likewise for the equivalent experiments with weaker wind forcing. Shifting the wind stress forcing north of Drake Passage does weaken the Drake Passage volume transport, most dramatically for the strongest wind forcing and only slightly for the weaker wind forcings, but a strong circumpolar current remains in all cases. For the most northerly profile, the Drake Passage volume transport actually increases slightly, due to the variation of the Ekman transport with the inverse Coriolis parameter.

The result that a large circumpolar transport remains when the wind is shifted north of Drake Passage is consistent with the earlier findings of Allison et al. (2010) for less extreme shifts. This raises questions about the applicability of mechanisms that have been proposed for the sensitivity of the past and future ocean to relatively subtle variations in the latitude of the southern hemisphere wind jet (e.g., Toggweiler et al, 2006; Fyfe et al., 2007; Le Quéré et al., 2007).

\section{What sets the Drake Passage volume transport?}

The Drake Passage volume transport is well approximated by:

$$
T_{d p}=\int_{d p} h u d y \approx-\frac{g_{r} h_{d p}^{2}}{2 f_{d p}},
$$

where the integral is evaluated across the model Drake Passage and $h_{d p}$ and $f_{d p}$ are evaluated at the northern edge of Drake Passage (where the streamlines are concentrated). In deriving (6.1), the velocity has been approximated as geostrophic and the layer thickness neglected to the south of Drake Passage. Note that $f_{d p}$ is negative and thus $T_{d p}$ is positive.

Following a similar approach to Allison et al. (2010), an approximate expression is now sought for the circumpolar transport in terms of the wind stress and model parameters. Combining $h / f \times$ the zonal component of (2.1) with the 
(a)

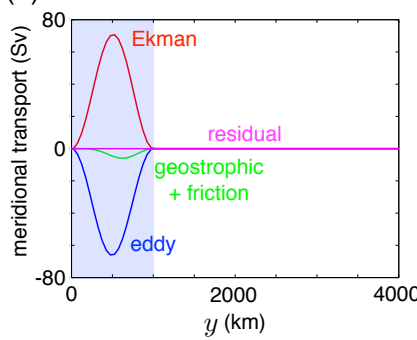

(b)

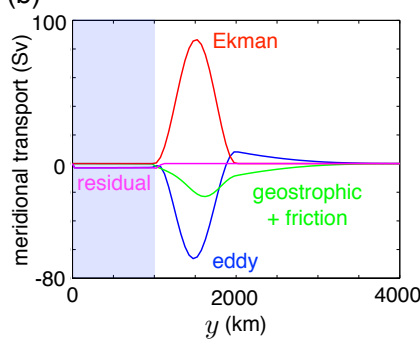

(c)

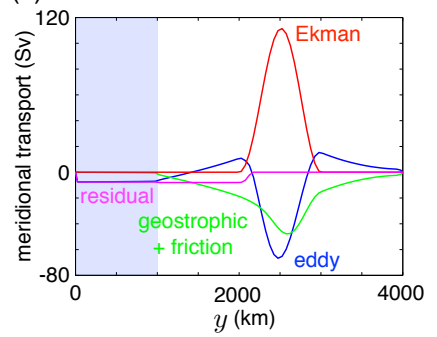

(d)

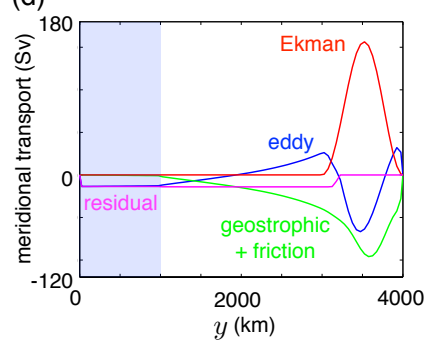

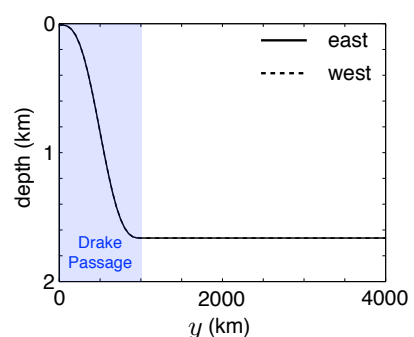
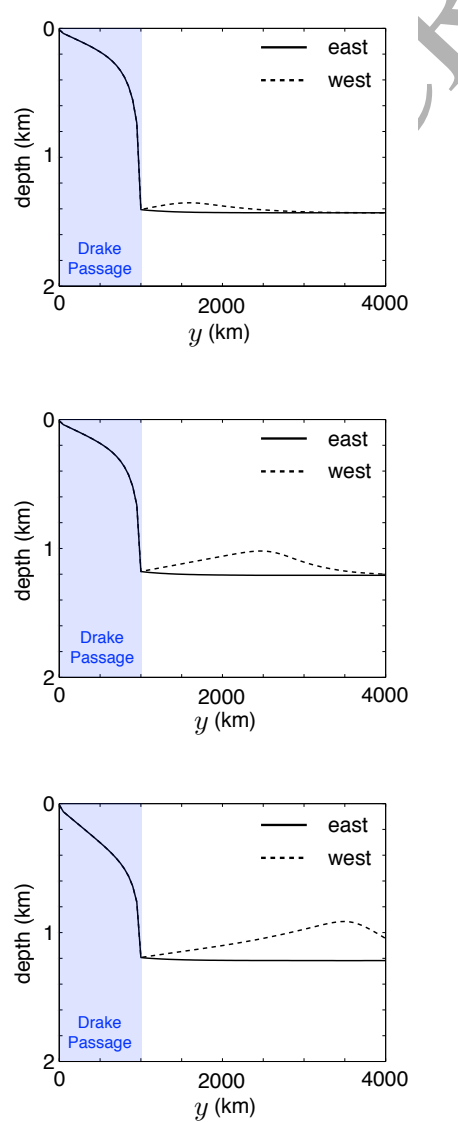

)

Figure 10: Net meridional volume transport decomposed into constituent terms, and the variation of the layer thickness, $h$, with meridional distance, $y$, along the eastern (solid lines) and western (dashed lines) boundaries, in solutions (a) W014, (a) W124, (a) W234, (a) W344. See the caption to Fig. 5 for more details. Note the different scales on the vertical axes of the left panels. 


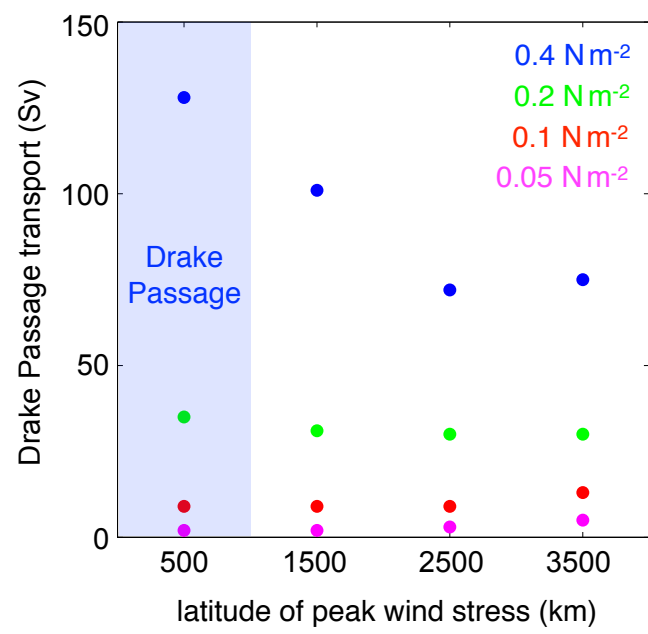

Figure 11: Variation of Drake Passage volume transport with the latitude of the wind jet for the narrow wind jet solutions (W01n, W12n, W23n, W34n; $n=0,1,2,4$ ). Also shown with light blue shading is the latitude band of the model Drake Passage.

meridional component of the eddy bolus transport, the northward transport velocity is

$$
h\left(v+v^{*}\right)=\frac{\partial}{\partial x}\left(\frac{g_{r} h^{2}}{2 f}\right)-\frac{\tau_{s}^{(x)}}{\rho_{0} f}-\left(\kappa_{g m}+c \delta_{s}\right) \frac{\partial h}{\partial y} .
$$

Equation (6.2) is integrated over the area bounded by the streamlines that touch the northern and southern extremes of the model Drake Passage, as sketched in Fig. 12. These streamlines are, in turn, approximated by the geostrophic streamlines (layer thickness contours), except close to the western boundary where, due to the pressure drop across the basin, it is necessary to connect the northernmost/ayer thickness contour to the western boundary as sketched in

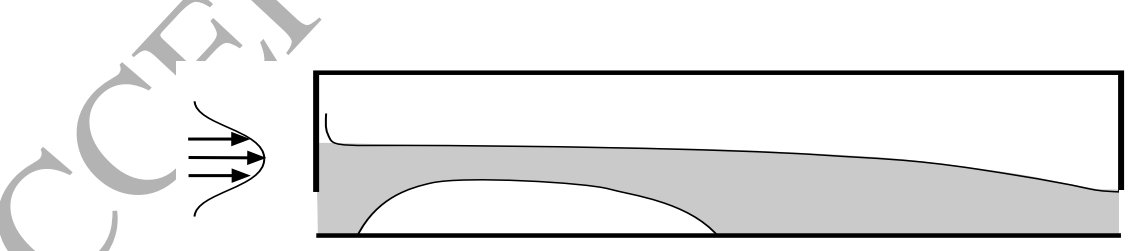

Figure 12: Schematic showing the shaded area over which (6.2) is integrated, bounded by the streamlines that touch the northern and southern extremes of the model Drake Passage. These streamlines are, in turn, approximated by the geostrophic streamlines (layer thickness contours), except close to the western boundary where, due to the pressure drop across the basin, it is necessary to connect the northernmost layer thickness contour to the western boundary. See the text for further details. 
Fig. 12 .

The result, neglecting the frictional term, is

$$
\iint h\left(v+v^{*}\right) d x d y \approx \int \frac{g_{r}}{2 f}\left(h_{e}^{2}-h_{w}^{2}\right) d y-\iint \frac{\tau_{s}^{(x)}}{\rho_{0} f} d x d y-\kappa_{g m} h_{d p} x_{0} .
$$

Assuming the left-hand-side of this equation approximately vanishes, which holds provided there is no residual northward transport (in which case a streamline cannot be defined), and neglecting the geostrophic term, a theoretical prediction is obtained for the layer thickness at the northern tip of Drake Passage,

$$
h_{d p} \approx \frac{1}{\kappa_{g m} x_{0}} \iint \frac{\tau_{s}^{(x)}}{\rho_{0} f} d x d y
$$

from which a prediction of $T_{d p}$ follows using (6.1). This is the result of Allison et al. (2010) that the circumpolar transport depends on the integral of the wind stress over the circumpolar streamlines. This prediction is tested against the diagnosed model values in the top panels of Fig. 13. In general, the integral wind stress serves as a useful predictor of the Drake Passage transport. However, there is some scatter and the predicted value generally exceeds the actual transport, by a considerable margin in the case of large wind stress, in particular when the wind jet is located far north of the model Drake Passage.

An improved prediction of the circumpolar transport can be obtained by retaining the geostrophic term,

$$
h_{d p} \approx \frac{1}{\kappa_{g m} x_{0}} \iint \frac{\tau_{s}^{(x)}}{\rho_{0} f} d x d y-\frac{1}{\kappa_{g m} x_{0}} \int \frac{g_{r}}{2 f}\left(h_{e}^{2}-h_{w}^{2}\right) d y .
$$

This is tested against the diagnosed model values in the central panels of Fig. 13. Note that most of the scatter is now removed, except for the cases in which the wind stress is located far north of the model Drake Passage.

In these latter cases, some of the discrepancy can be explained by noting that the residual transport across the model Drake Passage does not vanish (see Fig. 10). To obtain a further improved estimate, the residual transport can be integrated across the model Drake Passage and included in the result,

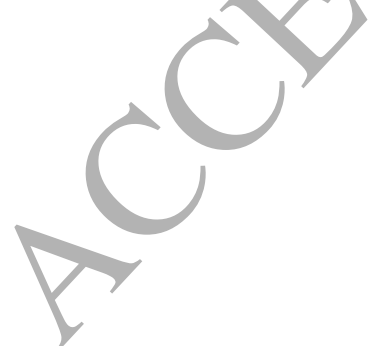

$$
\begin{aligned}
h_{d p} \approx & \frac{1}{\kappa_{g m} x_{0}} \iint \frac{\tau_{s}^{(x)}}{\rho_{0} f} d x d y \\
& -\frac{1}{\kappa_{g m} x_{0}} \int \frac{g_{r}}{2 f}\left(h_{e}^{2}-h_{w}^{2}\right) d y \\
& +\frac{1}{\kappa_{g m} x_{0}} \iiint_{d p} h\left(v+v^{*}\right) d x d y .
\end{aligned}
$$

${ }_{523}$ This is tested against the diagnosed model values in the lower panels of Fig. 13 ${ }_{524}$ where we see that yet more of the scatter is removed, except in the cases in which ${ }_{525}$ the wind stress is located at the northern extreme of the basin. The final term 


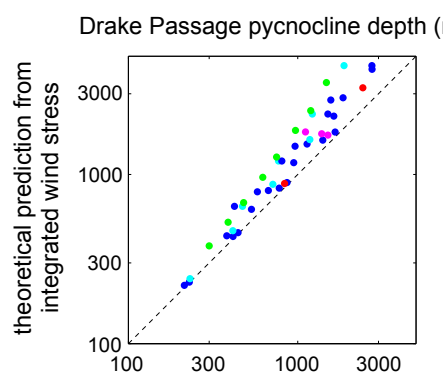

Drake Passage transport (Sv)
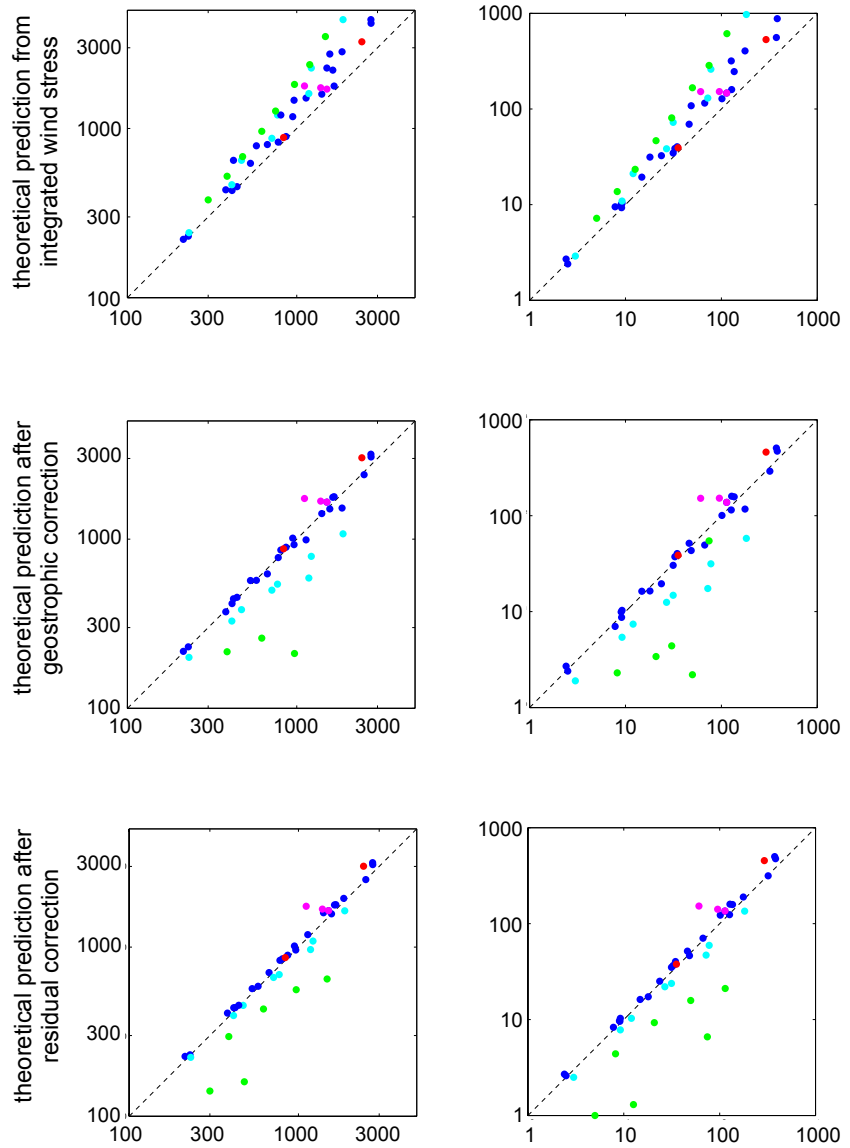

diagnosed from model

Figure 13: Comparison of the theoretical predictions of the pycnocline depth at the northern tip of "Drake Passage" (left panels) and the "Drake Passage" volume transport (right panels) against values diagnosed from the full model calculations. The three rows show the theoretical predictions obtained by: (i) integrating the zonal wind stress over the circumpolar streamlines; (ii) additionally correcting for the diagnosed northward geostrophic transport; (iii) further correcting for the diagnosed residual transport across Drake Passage. Blue points correspond to wind profiles where the peak wind stress lies at $y \leq L / 2$, cyan points where the peak wind stress lies in the range $L / 2<y<3 L / 4$, and green points where the wind stress lies at $y \geq 3 L / 4$. The three magenta points corresponds to the calculations in which the drag coefficient is increased (the control value lying on the dashed line), and the red points to the two additional calculations in which the Gent and McWilliams eddy diffusivity is increased and decreased.

in 6.6 neglects any residual transport north of Drake Passage, inclusion of which 
actually degrades the prediction (not shown) for reasons that remain unclear, but are probably related to the presence of a retroflection of the circumpolar current in the solutions concerned.

Finally, we note that an increased the linear drag coefficient leads to a reduction in Drake Passage transport. This effect can be incorporated into the theoretical prediction by replacing the eddy diffusivity, $\kappa_{g m}$ with $\kappa_{g m}+c \delta_{s}$ (assuming a mean value of $c$ ) and recalculating the integrals through the same procedure (not shown).

Thus, in summary, the integral of the wind stress over the circumpolar streamlines serves as a useful predictor of the Drake Passage transport in the model, even when the wind stress is located north of the model Drake Passage. The simplest measure proposed by Allison et al. (2010) generally overestimates the circumpolar transport. This can be improved substantially by correcting for basin-wide pressure gradients, and to a lesser extent for the residual volume transport across the model Drake Passage. Note that all of these "predictions" are implicit in the sense that one requires advance knowledge of the path of the circumpolar streamlines. However, in practice, these can often be anticipated from the latitude of the peak wind stress, although this relationship can break down when the latitude of the peak wind stress is not well defined (not shown).

In reality, the eddy diffusivity will vary strongly across the ACC and hence the main value of these predictions is pedagogical, in illustrating that there is a relation between the volume transport of the ACC and a (weighted) integral of the wind stress forcing, rather than any practical predictive skill.

\section{Concluding remarks}

In this manuscript we have formulated and analyzed solutions of a simple reduced-gravity model of the ACC. The model bears many similarities to that developed by Gill (1968) for a barotropic ocean, but with the model parameters reinterpreted in terms of quantities at the heart of contemporary descriptions of ACC dynamics, such as the pycnocline depth and Gent and McWilliams eddy diffusivity. Our main findings are:

- A substantial circumpolar volume transport is obtained when the latitude of the wind jet is shifted north of the model Drake Passage, even by several thousand kilometers. )

Meridional excursions of the modelled ACC are described by a linear vorticity balance between advection of planetary vorticity and stretching by the residual of the Ekman and eddy-induced upwellings.

- The integral of the wind stress over the circumpolar contours is a useful predictor of the magnitude of the volume transport through the model Drake Passage, although it is necessary to correct for basin-wide zonal pressure gradients in order to obtain good quantitative agreement. 
We hope this work will restore Gill (1968) to its rightful place at the center-stage of theoretical understanding of the ACC.

These results have significant implications for studies of past and future climate change that assume a relation between the latitude of the southern hemisphere wind jet and the circulation along and/or across the ACC (e.g., Toggweiler et al., 2006; Fyfe et al., 2007; Le Quéré et al., 2007). In particular, special emphasis is often placed on the wind stress across the circumpolar latitude band, or at the northern tip of Drake Passage. While our model solutions do show variations in circumpolar volume transport as the wind jet is shifted northward, a strong circumpolar volume transport remains even when the wind jet is entirely north of the circumpolar latitudes. It remains to be established whether this result extends to the overturning circulation across the ACC which, for example, is more important for ocean carbon uptake. Nevertheless the results reported here do suggest that current thinking on these topics may be over-simplistic.

While the model has proved valuable in addressing some zero-order questions, it has many limitations. An obvious extension is to multiple layers (e.g., Bell, 2015), particularly in the light of the result that the model selects, through its dynamics, regions of outcropping and dense water formation. A natural question is to what extent is a multi-layer model able to predict the lateral structure, including the formation sites, of the Antarctic intermediate and bottom water masses through a finite residual circulation across the ACC (Marshall, 1997; Marshall and Radko, 2003) - a "ventilated thermocline" model for the Southern Ocean (cf. Luyten et al., 1983). It is clear that such a model will require a more realistic representation of buoyancy forcing.

A further issue that we have glossed over is the role of the barotropic mode. Firstly, while the eddy-induced upwelling might modify "Sverdrup balance" within a reduced-grayity layer, it cannot affect the vorticity budget of the entire fluid column, at least not directly. Thus, implicit in our reduced-gravity solutions is an opposite meridional volume transport in the abyssal ocean (albeit over an infinite depth, so not affecting the path of the surface streamlines). In practice, bottom topography will significantly modify the depth-integrated vorticity budget (e.g., Marshall, 1995a,b; Hughes and Killworth, 1995; Hughes, 2005) so such concerns may be of limited practical significance. Secondly, Rossby waves propagate eastward in the core of the ACC (Hughes et al., 1998; Hughes, 2005), Doppler-shifted by the depth-mean velocity (Klocker and Marshall, 2014). Since westward Rossby propagation plays a role in establishing the structure of the present solutions, it is natural to ask how the solution is modified when the Rossby waves are Doppler-shifted and propagate eastward.

Finally, we wish to reiterate that all of the present results have been obtained using a model with parameterized eddies. The ACC exhibits far less sensitivity to changes in wind stress forcing in models with explicit, rather than parameterized, eddies (Hallberg and Gnanadesikan, 2001; Tansley and Marshall, 2001b; Hallberg and Gnanadesikan, 2006; Hogg and Blundell, 2006; Meredith and Hogg, 2006; Farneti et al., 2010; Farneti and Delworth, 2010; Munday et al., 2013). In addition, eddy activity becomes enhanced in the lee of major topo- 
graphic features, also leading to the formation of inertial jets (e.g., MacCready and Rhines, 2001; Tansley and Marshall, 2001a; Abernathey and Cessi, 2014). Hence it remains to be seen how the present results apply to an ocean with explicit eddies.

\section{Acknowledgements}

We are grateful to Mike Bell, Paola Cessi, James Maddison, Wilbert Weijer and the anonymous reviewers for suggestions that led to an improved manuscript. Financial supported was provided by the U.K. Natural Environment Research Council (NE/H005668/1). HLJ was supported by a Royal Society University Research Fellowship.

\section{Appendix. Method of solution}

The time scale for a time-dependent reduced gravity model of the ACC to equilibrate is several millennia (Allison et al., 2011). Thus for computational efficiency equilibrium solutions to (2.1-2.3) are obtained through a relaxation method. The grid spacing in the basin interior is a uniform $50 \mathrm{~km}$. Variable grid spacing is employed in $x$ near the meridional boundaries to enhance resolution within the boundary layers, as sketched in Fig. 14. Thus, approaching the boundary over the last 14 grid cells, each grid spacing is roughly a factor 0.75 smaller than its neighbor. These 14 cells are equivalent to 3 grid cells in the basin interior, the finest grid spacing being $0.9 \mathrm{~km}$ adjacent to the boundaries. To maintain a structured grid, the same grid spacings are also applied within the Drake Passage latitude band.

We rewrite (2.2) as a finite-difference diffusion equation on a C-grid with a forward Euler time step:

$$
h^{\text {new }}=h^{\text {old }}-\left(\nabla \cdot \mathbf{U}-w_{e k}\right) \Delta t(x, y)
$$

Here the layer thickness flux excludes the Ekman contribution but includes the eddy bolus transport:

$$
\mathbf{U}=\frac{g_{r}}{2 f} \mathbf{k} \times \nabla{\overline{h^{2}}}^{x y}-\kappa_{g m} \nabla h-\frac{r g_{r}}{2 f^{2}} \nabla h^{2}
$$

${ }_{641}$ where $\bar{h}^{x y}$ indicates an average of $h$ between the four adjacent points. The first term on the right-hand side can be decomposed into a westward Rossby flux and a dynamically-inert rotational flux, as in (2.4), but we retain the form in (A.2) for consistency with the boundary condition discussed below. The time-step is allowed to vary spatially and is chosen to ensure both stability and efficient convergence:

$$
\Delta t(x, y)=\frac{1}{8\left(\kappa_{g m}+c \delta_{s}\right)}\left(\frac{1}{\Delta x^{2}}+\frac{1}{\Delta y^{2}}\right)^{-1} .
$$

The northern half-width boundary cells, shaded grey in Fig. 14, are treated through a separate, two-step procedure. To understand the rationale for this, 


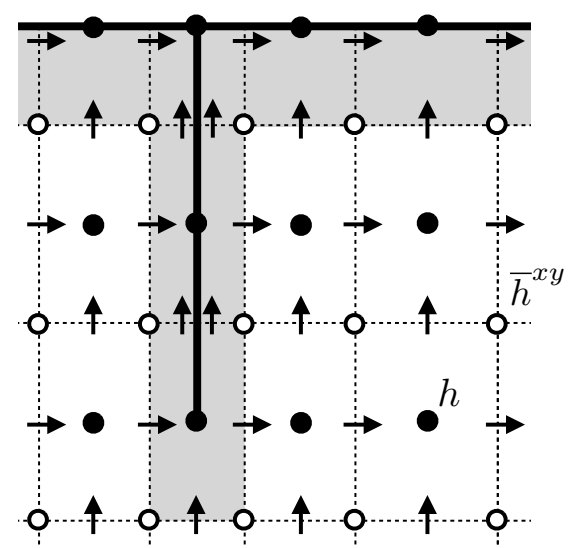

Figure 14: The equations (A.1), (A.2) and (A.4) are solved on a C-grid with a default grid spacing of $50 \mathrm{~km}$, reducing smoothly to $1 \mathrm{~km}$ to enhance resolution within the western and eastern boundary layers. Adjacent to the boundaries is a line of half-width cells (shaded) such that the layer thickness, $h$ is located on the boundaries. Also shown are the zonal and meridional transports, $U$ and $V$, as defined in (A.2), and the averaged layer thickness, $\bar{h}^{x y}$, required for computation of the geostrophic transports

note that the advective transport on the boundary can be simplified using the boundary condition (2.3):

$$
\mathbf{U}_{\perp}=0, \quad \mathbf{U}_{\|}=\nsucc \frac{r g_{r}}{2 f^{2}}\left(1+\frac{f^{2}}{r^{2}}\right) \nabla_{\|} h^{2} .
$$

Thus, the effect of the boundary condition is equivalent to an along-boundary diffusion that can be shown to be a factor $\left(c \delta_{s} / \kappa_{g m}\right) f^{2} / r^{2}$ larger than in the basin interior; for the majority of the model calculations reported here, this factor is roughly $10^{5}$. The effect of this rapid along-boundary diffusion is to rapidly remove unbalanced along-boundary pressure gradients and, in conjunction with the thickness flux into the boundary grid cells through (A.2), can be shown to give rise to Kelvin waves (or their low-frequency counterparts; Marshall and Johnson, 2013).

For computational efficiency, we first update the mean layer thickness across all of the boundary cells shaded grey in Fig. 14. This is carried out with a time step that is determined empirically to be 20 times that given by (A.3) for one of the northern cells. We then update for the variation of the layer thickness along the boundary with a time step given by (A.3), except replacing the diffusion coefficient by its enhanced along-boundary value.

Each equilibrium solution is obtained by stepping forward (A.1) through a minimum of 60000 iterations, where necessary repeating this process until convergence is achieved. To give an idea of the efficiency of the above procedure, the equilibrium solution is achieved after the equivalent of 10 model years of integration of the time-dependent reduced-gravity equations with a uniform grid 

ancy conditions on a circumpolar current. Geophys. Res. Lett. 34, L14605, doi:10.1029/2007GL030379.

${ }_{02}$ Fyfe, J. C., Saenko, O. A., Zickfeld, K., Eby, M., Weaver, A. J., 2007. The role 703 of poleward-intensifying winds on Southern Ocean warming. J. Climate 20, $704 \quad 5391-5400$. 
735 Hüghes, C. W., 2005. Nonlinear vorticity balance of the Antarctic Circumpolar ${ }_{736}$ Current. J. Geophys. Res. 110, C11008, doi:10.1029/2004JC002753.

Hughes, C. W., Jones, M. . S., Carnochan, S., 1998. Use of transient features to identify eastward currents in the Southern Ocean. J. Geophys. Res. 103, 2929-2942.

Gautschi, W., 1964. Error function and Fresnel integrals. In: Abramowitz, M., Stegun, I. A. (Eds.), Handbook of Mathematical Functions. United States Department of Commerce, pp. 295-330.

Gent, P. R., McWilliams, J. C., 1990. Isopycnal mixing in ocean circulation models. J. Phys. Oceanogr. 20, 150-155.

Gent, P. R., Willebrand, J., McDougall, T. J., McWilliams, J. C., 1995. Parameterizing eddy-induced tracer transports in ocean circulation models. J. Phys. Oceanogr. 25, 463-474.

Gill, A. E., 1968. A linear model of the Antarctic Circumpolar Current. J. Fhiid Mech. 32, 465-488.

Gnanadesikan, A., 1999. A simple predictive model of the structure of the oceanic pycnocline. Science 283, 2077-2081.

Gnanadesikan, A., Hallberg, R. W., 2000. On the relátionship of the circumpolar current to southern hemisphere winds in coarse-resolution ocean models. J. Phys. Oceanogr. 30, 2013-2034.

Green, J. S., 1970. Transfer properties of the large-scale eddies and the general circulation of the atmosphere. Quart. J Roy. Meteor. Soc. 96, 157-185.

Hallberg, R. W., Gnanadesikan, A., 2001. An exploration of the role of transient eddies in determining the transport of a zonally reentrant current. J. Phys. Oceanogr. 31, 3312-3330.

Hallberg, R. W., Gnanadesikan, A., 2006. The role of eddies in determining the structure and response of the wind-driven southern hemisphere overturning: Results from the Modeling Eddies in the Southern Ocean (MESO) project. J. Phys. Oceanogr. 36, 2232-2252.

Hogg, A. M., 2010. An Antarctic Circumpolar Current driven by surface buoyancy forcing. Geophys. Res. Lett. 37, L23601, doi:10.1029/2010GL044777.

Hogg, A. M., Blundell, J. R., 2006. Interdecadal variability of the Southern Ocean. J. Phys. Oceanogr. 36, 1626-1645.

Hughes, C. W., 1997. Comments on "On the obscurantist physics of 'form drag' in theorizing about the circumpolar current". J. Phys. Oceanogr. 27, 209-210. 
Marshall, D. P., Johnson, H. L., 2013. Propagation of meridional circulation 770 anomalies along western and eastern boundaries. J. Phys. Oceanogr. 43, 26992717 .

Marshall, J., Radko, T., 2003. Residual-mean solutions for the Antarctic Circumpolar Current and its associated overturning circulation. J. Phys. Oceanogr. 33, 2341-2354.

Hughes, C. W., Killworth, P. D., 1995. Effects of bottom topography in the large-scale circulation of the Southern Ocean. J. Phys. Oceanogr. 25, 24852497.

(1994. Effects of partial meridional barriers on the Antarctic Circumpolar Current - wind driven barotropic model. Dyn. Atmos. Oceans 20, $315-341$.

Johnson, G. C., Bryden, H. L., 1989. On the size of the Antarctic Circumpolar Current. Deep Sea Res. 36, 39-53.

Jones, D. C., Ito, T., Lovenduski, N., 2011. The transient response of the Southern Ocean pycnocline to changing atmospheric winds. Geophys. Res. Lett. 38, L15604, doi:10.1029/2011GL048145.

Klocker, A., Marshall, D. P., 2014. Advection of baroclinic eddies by depth mean flow. Geophys. Res. Lett. 41, 3517-3521.

LaCasce, J. H., Isachsen, P. E., 2010. The linear models of the ACC. Prog. Oceanogr. 84, 139-157.

Le Quéré, C., Rödenbeck, C., Buitenhuis, E., Conway, T., Langenfelds, R., Gomez, A., Labuschagne, C., Ramonet, M., Nakazawa, T., Metzl, N., Gillett, N., Heimann, M., 2007. Saturation of the Southern Ocean $\mathrm{CO}_{2}$ sink due to recent climate change. Science 316, 1735-1738.

Luyten, J. R., Pedlosky, J., Stommel, H., 1983. The ventilated thermocline. J. Phys. Oceanogr. 13, 292-309.

MacCready, P., Rhines, P. B., 2001. Meridional transport across a zonal channel: Topographic localization. J. Phys. Oceanogr. 31, 1427-1439.

Marshall, D., 1995a. Influence of topography on the large-scale ocean circulation. J. Phys. Oceanogr. 25, 1622-1635.

Marshall, D., 1995b. Topographic steering of the Antarctic Circumpolar Current. J. Phys. Oceanogr. 25, 1636-1650.

Marshall, D., 1997. Subduction of water masses in an eddying ocean. J. Mar. Res. 55, 201-222.

Mazloff, M. R., Heimbach, P., Wunsch, C., 2010. An eddy-permitting Southern Ocean state estimate. J. Phys. Oceanogr. 40, 880-899. 
Rintoul, S. R., Hughes, C., Olbers, D., 2001. The Antarctic Circumpolar Current system. In: Siedler, S., Church, J., Gould, J. (Eds.), Ocean Circulation and Climate, 1st Edition. Academic Press, pp. 271-302.

Russell, J. L., Stouffer, R. J., Dixon, K. W., 2006. Intercomparison of the Southern Ocean circulations in IPCC coupled model control simulations. J. Clim. 19, 4560-4575.

Samelson, R. M., 2011. Time-dependent adjustment in a simple model of the mid-depth meridional overturning cell. J. Phys. Oceanogr. 41, 1009-1025. 
Stommel, H., 1948. The westward intensification of wind-driven ocean currents. Trans. Am. Geophys. Union 29, 202-206.

Stommel, H., 1957. A survey of ocean current theory. Deep Sea Res. 4, 149-184.

Stone, P., 1972. A simplified radiative-dynamical model for the static stability of the rotating atmosphere. J. Atmos. Sci. 29, 405-418.

Straub, D., 1993. On the transport and angular momentum balance of channel models of the Antarctic Circumpolar Current. J. Phys. Oceanogr. 23, 776-782.

Sverdrup, H. U., 1947. Wind-driven currents in a baroclinic ocean; with application to the equatorial currents of the eastern pacific. Proc Nat. Acad. Sci. U.S. $22,318-326$.

Tansley, C. E., Marshall, D. P., 2001a. Flow past a cylinder on a $\beta$ plane, with application to Gulf Stream separation and the Antarctic Circumpolar Current. J. Phys. Oceanogr. 31, 3274-3283.

Tansley, C. E., Marshall, D. P., 2001b. On the dynamics of wind-driven circumpolar currents. J. Phys. Oceanogr. 31, 3258-3273.

Toggweiler, J. R., Russell, J. L., Carson, S. R., 2006. Midlatitude westerlies, atmospheric $\mathrm{CO}_{2}$, and climate change during the ice ages. Paleoceanography 21, PA2005, doi:10.1029/2005PA001154.

Vallis, G. K., 2000. Large-scale circulation and production of stratification: effects of wind, geometry and diffusion. J. Phys. Oceanogr. 30, 933-954. 